\title{
Angiopoietin-2 exacerbates cardiac hypoxia and inflammation after myocardial infarction
}

\author{
Seung-Jun Lee, ${ }^{1}$ Choong-kun Lee, ${ }^{1,2}$ Seok Kang, ${ }^{1,2}$ Intae Park, ${ }^{1,2}$ Yoo Hyung Kim, ${ }^{1,2}$ Seo Ki Kim, ${ }^{1,2}$ Seon Pyo Hong, ${ }^{1,2}$ \\ Hosung Bae, ${ }^{1,2}$ Yulong He, ${ }^{3}$ Yoshiaki Kubota, ${ }^{4}$ and Gou Young Koh ${ }^{1,2}$ \\ 'Center for Vascular Research, Institute for Basic Science, Daejeon, South Korea. '2Graduate School of Medical Science and Engineering, Korea Advanced Institute of Science and Technology (KAIST), \\ Daejeon, South Korea. ${ }^{3}$ Cyrus Tang Hematology Center, Soochow University, Suzhou, China. ${ }^{4}$ The Laboratory of Vascular Biology, School of Medicine, Keio University, Tokyo, Japan.
}

\begin{abstract}
Emerging evidence indicates that angiopoietin-2 (Angpt2), a well-recognized vascular destabilizing factor, is a biomarker of poor outcome in ischemic heart disease. However, its precise role in postischemic cardiovascular remodeling is poorly understood. Here, we show that Angpt2 plays multifaceted roles in the exacerbation of cardiac hypoxia and inflammation after myocardial ischemia. Angpt2 was highly expressed in endothelial cells at the infarct border zone after myocardial infarction (MI) or ischemia/reperfusion injury in mice. In the acute phase of MI, endothelial-derived Angpt2 antagonized Angpt1/Tie2 signaling, which was greatly involved in pericyte detachment, vascular leakage, increased adhesion molecular expression, degradation of the glycocalyx and extracellular matrix, and enhanced neutrophil infiltration and hypoxia in the infarct border area. In the chronic remodeling phase after MI, endothelial- and macrophage-derived Angpt2 continuously promoted abnormal vascular remodeling and proinflammatory macrophage polarization through integrin $\alpha_{5} \beta_{1}$ signaling, worsening cardiac hypoxia and inflammation. Accordingly, inhibition of Angpt2 either by gene deletion or using an antiAngpt2 blocking antibody substantially alleviated these pathological findings and ameliorated postischemic cardiovascular remodeling. Blockade of Angpt2 thus has potential as a therapeutic option for ischemic heart failure.
\end{abstract}

\section{Introduction}

Ischemic heart disease is the leading cause of death worldwide, causing about 8.8 million deaths in 2015 and accounting for approximately $15.5 \%$ of all deaths that year (1). Prompt revascularization by percutaneous coronary intervention or bypass surgery markedly reduces acute mortality from myocardial infarction (MI); however, increased morbidity due to ischemic heart failure has become a global burden (2), highlighting the need for additional therapeutic modalities to alleviate postischemic cardiovascular remodeling.

Evidence indicates that endothelial dysfunction is a key component of the pathophysiology of heart failure $(3,4)$. VEGF-A, referred to herein as VEGF, is highly expressed in myocardial cells. While VEGF helps preserve the cardiac capillary bed under homeostatic conditions, it acts as a stimulating factor for angiogenesis, vascular leakage, and inflammation under ischemic conditions (5-11). VEGF is downregulated during the transition from compensated hypertrophy to decompensated heart failure, reducing its capacity to preserve the cardiac capillary bed or to generate new vessels in response to cardiac hypertrophy (3). Although a multitude of animal studies have shown that VEGF induces angiogenesis in the ischemic myocardium and that it mitigates adverse remodeling, to date, clinical trials of VEGF have not yet demonstrated any clinical benefits (5). This discrepancy is likely due to the negative effect of VEGF on the ischemic heart. Indeed,

Conflict of interest: The authors have declared that no conflict of interest exists. License: Copyright 2018, American Society for Clinical Investigation.

Submitted: January 5, 2018; Accepted: August 21, 2018.

Reference information: J Clin Invest. 2018;128(11):5018-5033.

https://doi.org/10.1172/JCI99659.
VEGF exacerbates tissue damage after MI (6) and ischemic stroke (7) by inducing the disintegration of endothelial cells (ECs), thereby aggravating vascular leakage, inflammation, and hypoxia (8). Inhibiting VEGF-induced vascular permeability can reduce tissue edema and improve outcome in MI (8) and stroke (7). However, due to the angiogenic and cytoprotective effects of VEGF (9), VEGF inhibition after MI confronts a major hurdle that can exacerbate ischemia and therefore tissue damage.

Angiopoietins, the other family of major angiogenic growth factors, regulate vascular maturation and stability by binding to the endothelial cell-specific (EC-specific) Tie2 receptor and to subtypes of integrins $(10,11)$. Angiopoietin-1 (Angpt1) is an agonistic ligand of Tie 2 that maintains the quiescence and integrity of ECs (12). In contrast, Angpt 2 is a partial Tie2 antagonist or agonist that can modulate Angpt1/Tie2 signaling in context-dependent (10) and organ-specific manners (13-16). Hypoxia and inflammation induce Angpt2 expression on ECs (17), which destabilizes endothelial integrity (18). Although the deleterious effects of Angpt2 in augmenting vascular permeability and inflammation have been intensely investigated in sepsis and tumor angiogenesis (14), the precise role of Angpt2 in ischemic heart disease remains poorly elucidated. Emerging reports show that Angpt2 is associated with a greater risk of all-cause and cardiovascular mortality in the general population (19), with the deterioration of cardiac function in heart failure patients (20), and with higher mortality in MI and cardiogenic shock patients (21, 22). Given these meaningful findings, we investigated the role of Angpt2 in cardiac ischemia using mouse models. Our findings demonstrate that Angpt2 substantially exacerbates postischemic cardiovascular remodeling, including pericyte detachment, adhesion mole- 
A
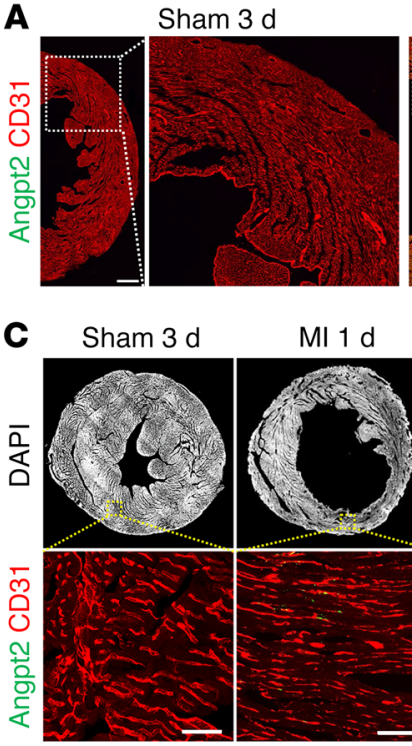

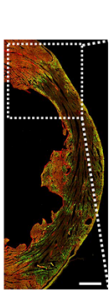

MI 3 d

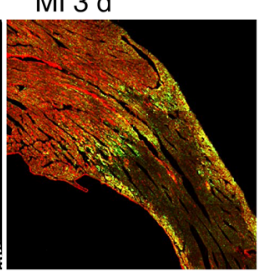

Ml 2 d

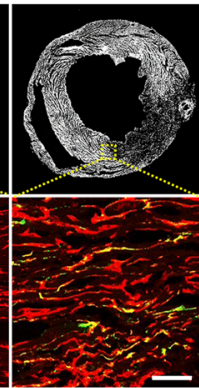

B

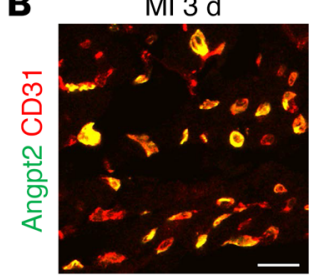

MI $3 \mathrm{~d}$

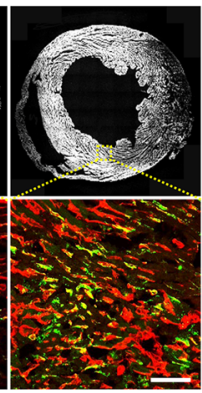

MI $7 d$

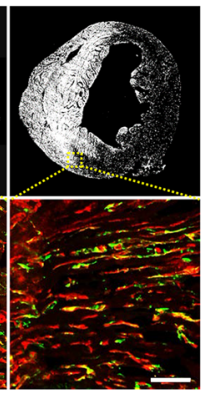

$\mathbf{F}$

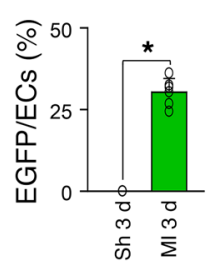

D

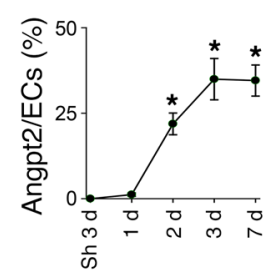

Figure 1. Angpt2 is highly expressed in ECs of the border zone after MI. Adult WT mice were subject to MI or sham procedure (Sh), hearts were harvested at indicated time points, and indicated molecules in heart sections were detected by immunostaining. (A and $\mathbf{B}$ ) Images for Angpt2 in ECs at the infarct border. Scale bars: $500 \mu \mathrm{m}$ (A); $20 \mu \mathrm{m}$ (B). (C and D) Temporal changes of Angpt2 in border zone ECs at indicated day after MI. $n=6$, each time point. Each box region is magnified below. Scale bars: $100 \mu \mathrm{m} .{ }^{*} P<0.05$ versus sham, Mann-Whitney $U$ test. (E and F) Images and comparisons of Angpt2 expression in ECs at the infarct border of Angpt2-EGFP mice. Box region is magnified at right. $n=5$, each group. Scale bars: $100 \mu \mathrm{m} .{ }^{*} P<0.05$ versus sham, Mann-Whitney $U$ test. Error bars represent mean $\pm \mathrm{SD}$.
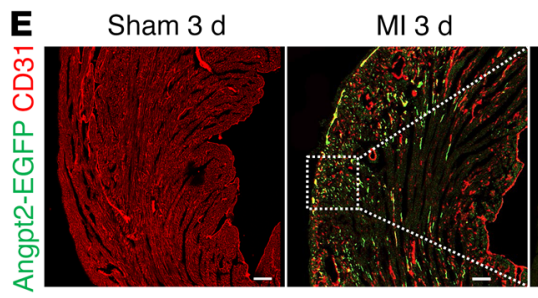

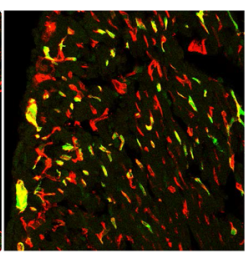

cule expression, glycocalyx and extracellular matrix (ECM) degradation, proinflammatory macrophage polarization, and adverse vascular remodeling. These findings identify Angpt 2 as a potential promising therapeutic target in ischemic heart disease, and antibody-mediated inhibition of Angpt2 ameliorates cardiac hypoxia and inflammation after MI.

\section{Results}

Angpt2 is highly expressed in ECs at the infarct border zone after MI. To determine the expression profile of Angpt2 in mouse heart after MI, we utilized a specific anti-Angpt2 antibody (23). High Angpt2 expression was observed in the infarct border zone 3 days after MI, while no Angpt2 expression was observed in shamoperated hearts (Figure 1A). A high-magnification view showed that Angpt2 expression was confined mainly to ECs at the border zone (Figure 1B). Sequential observations of ECs in the expanding infarct border revealed that Angpt2 expression began on day 2 , peaked on day 3 , and was maintained at the peak level until day 7 after MI (Figure 1, C and D). In vivo reporter gene analysis using Angpt2-EGFP mice confirmed that the increased Angpt2 expression was confined to the ECs (Figure 1, E and F). To further determine the identity of ECs expressing Angpt2, we utilized endothelial-lineage tracing mouse (VE-cadherin-Cre-ER ${ }^{\mathrm{T} 2}$ / Rosa-tdTomato, Supplemental Figure 1A; supplemental material available online with this article; https://doi.org/10.1172/ JCI99659DS1) and a marker for circuiting ECs (CD117) (24). Lineage-tracing analysis revealed that Angpt 2 is almost exclusively expressed in ECs residing in the heart, rather than circulating ECs expressing CD117 (Supplemental Figure 1, B and C).
FOXO1 is an upstream regulator of Angpt2 expression in ECs of the border zone. We next investigated the spatiotemporal expression of forkhead box protein O1 (FOXO1), an upstream transcriptional regulator of Angpt2 expression in the ECs after MI (25). In the sham-operated control mouse heart, neither ECs nor cardiomyocytes (CMs) expressed FOXO1 (Figure 2A). In contrast, there was a striking increase in FOXO1 expression, which is known to protect $\mathrm{CMs}$ against ischemic insult through regulation of antioxidant genes, in CMs in the infarct border area of MI mice (26). This increase was distinct 1 day after MI and then rapidly decreased (Figure 2, A and B). Of particular note, FOXO1 expression was robustly increased and showed nuclear or nucleocytoplasmic localization in ECs at the border zone 2 days after MI, which correlates with the Angpt2 expression profile (Figure 2, A and B). Immunoblot analysis demonstrated a consistent finding of increased FOXO1 expression after MI (Figure 2, C and D). Indeed, concurrent Angpt2 expression and FOXO1 nuclear localization were observed in the ECs (Figure 2E). To investigate whether FOXO1 directly regulated Angpt2 expression in ECs, we generated Foxo $1^{\text {iEEC }}$ mice by crossing Foxo $1^{\mathrm{fl} / \mathrm{fl}}$ mice (27) with VE-cadherin-Cre-ER ${ }^{\mathrm{T} 2}$ mice (28) (Figure 2F), which depleted endothelial FOXO1 by approximately 85\% (Supplemental Figure 2, A-C). EC-specific deletion of Foxo1 using Foxo ${ }^{\mathrm{i} \triangle E C}$ mice markedly reduced Angpt2 expression in ECs (Figure 2, G and H). Taken together, these findings indicate that FOXO1 is a major transcriptional upstream regulator of Angpt2 expression in the ECs of murine ischemic hearts.

Angpt2 induces pericyte detachment from ECs of infarct border zone through antagonizing Angpt1/Tie2 signaling. Angpt2 is characterized by its context-dependent mode of action, which functions 
A

A Sham $2 d$

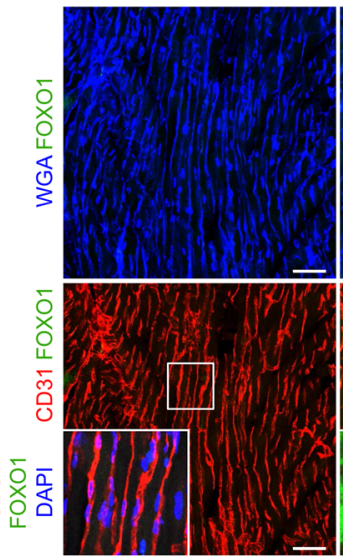

B

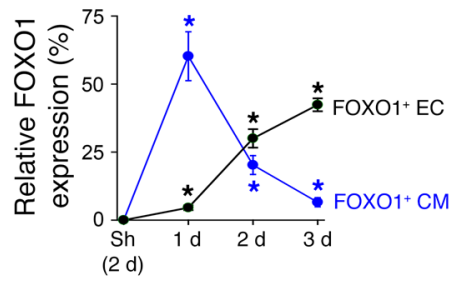

$\mathbf{E}$

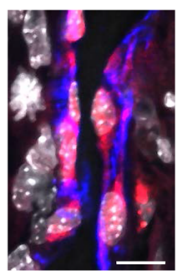

FOXO1 CD31 DAPI

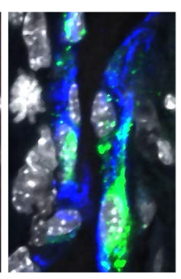

Angpt2 CD31 DAP
Ml $1 \mathrm{~d}$

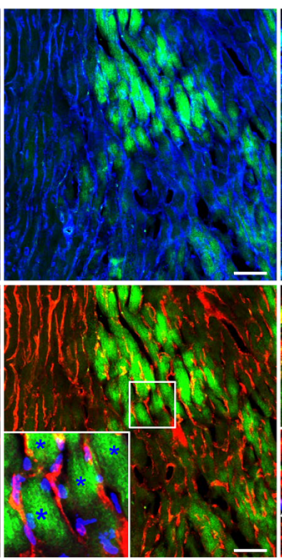

C

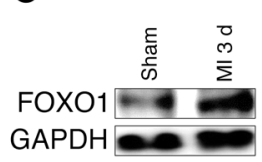

MI $2 \mathrm{~d}$

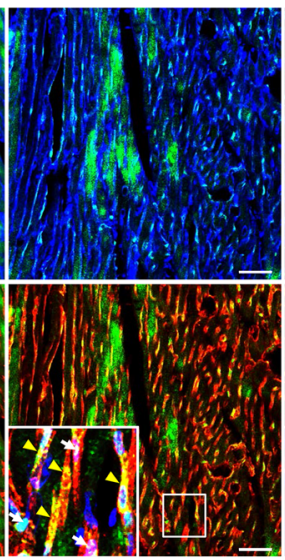

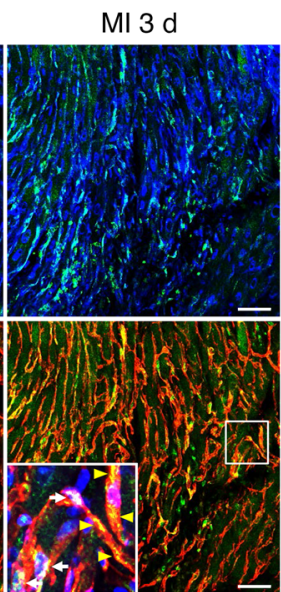

D

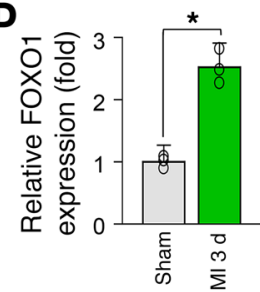

F Foxo1 ${ }^{\text {iSEC }}$ mouse

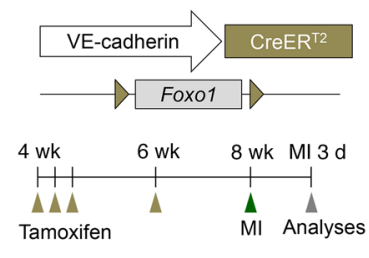

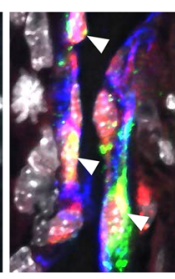

FOXO1 Angpt2 CD31 DAPI
G

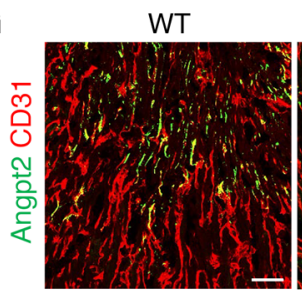

Foxo1 ${ }^{i \Delta E C}$

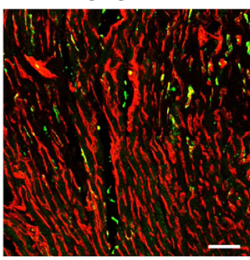

H

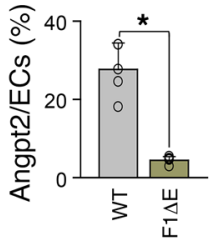

Figure 2. Marked increase of FOX01 governs Angpt2 expression in ECs of the infarct border after MI. Adult WT or Foxo ${ }^{\text {iUEC }}$ mice were subject to $\mathrm{Ml}$ or sham procedure, hearts were harvested at indicated time points, and indicated molecules in heart sections were detected by immunostaining. CM borders are highlighted by wheat germ agglutinin (WGA) staining. (A) Temporal changes of expression and distribution of FOXO1 after MI. Note rapidly increased FOXO1 in CMs (blue asterisks) at day 1 after $\mathrm{MI}$ and prominent nuclear (white arrows) or nucleocytoplasmic (yellow arrowheads) localization of FOXO1 in ECs at day 2 after MI. Each box region is magnified in left corner. Scale bars: $50 \mu \mathrm{m}$. (B) Comparisons of relative $\mathrm{FOXO1}$ expression in $\mathrm{CMs}$ and ECs after MI. $n=4-5$, each time point. ${ }^{*} P<$ 0.05 versus sham, Mann-Whitney $U$ test. (C and D) Immunoblot and densitometric analyses of indicated proteins at the infarct border after MI. Note increased expression of FOXO1 after MI. $n=3$, each group. ${ }^{*} P<0.05$ versus sham, Mann-Whitney $U$ test. (E) Images representing nuclear localization of FOXO1 in Angpt2+ border zone ECs (white arrowheads) at 3 days after MI. Scale bar: $20 \mu \mathrm{m}$. (F) Diagram depicting generation of FOXO1 ${ }^{\Delta E C}$ mice and experiment schedule. ( $\mathbf{G}$ and $\mathbf{H}$ ) Images and comparisons of Angpt2 expression in the ECs of WT and Foxo ${ }^{\text {ISECC }}(\mathrm{F} 1 \Delta \mathrm{E})$ mice. $n=5$, each group. Scale bars: $50 \mu \mathrm{m} .{ }^{*} P<0.05$ versus WT, Mann-Whitney $U$ test. Error bars represent mean \pm SD. as a Tie 2 agonist in the absence of Angpt1, but as an antagonist in the presence of Angpt1 (14). Therefore, we investigated the expression of Angpt1 in the heart using Angpt1-GFP reporter mice (29). In sham-operated control mice, Angpt1 expression marked by GFP fluorescence was found to be heterogeneous, but distinctly expressed throughout the myocardium, but the expression was not apparently changed at 3 days after MI in surviving myocardium (Figure 3, A and B). To evaluate the impact of Angpt1 and Angpt2 on Tie2 phosphorylation in ECs at the border zone, we generated Angpt $1^{i / / \Delta}$ mice by crossing Angpt $1^{f l / l}$ mice (29) with UBC-Cre-ER ${ }^{\mathrm{T} 2}$ mice and Angpt ${ }^{i \Delta E C}$ mice by crossing Angpt $2^{f / / f l}$ mice (27) with VE-cadherin-Cre-ER ${ }^{\mathrm{T} 2}$ mice (28) (Figure $3 \mathrm{C}$ ). Littermates that were Cre-ER ${ }^{\mathrm{T} 2}$ positive but flox/flox negative were defined as WT mice. Given the organ-specific role of Anpgt2 in the development and maturation of the vasculature, such as the vasculature of the kidney and Schlemm's canal of the eye $(14,15,30-32)$, we investigated the systemic vascular function (Supplemental Figure 3, A and B) and glomerular function (Supplemental Figure 3C), which showed no significant differences between the Angpt $2^{i \Delta E C}$ and WT mice. Heart ECs of sham-operated mice demonstrated robust Tie2 phosphorylation, which was markedly reduced after MI (Figure 3, D and E). Of note, the reduction in Tie2 phosphorylation in ECs after MI was further exacerbated in Angpt $1^{i / 4}$ mice, but was markedly mitigated in Angpt $2^{i \Delta E C}$ mice (Figure 3, D and E). These findings imply that Angpt2 acts as a Tie2 antagonist in the ischemic heart. Given that Angpt2-induced Tie2 inactivation causes pericytes to detach from ECs in inflammatory conditions $(14,25,30)$, we examined the ratio of pericyte coverage onto ECs of the border zone. As shown in Figure 3, F and G, the ECs with high Angpt2 expression had 55\% less pericyte coverage than the ECs with no or low Angpt2 expression. Consistently, pericyte detachment from ECs of the border zone at 3 days after MI was reduced by $59 \%$ in Angpt $2^{i \Delta E C}$ mice compared with WT mice (Figure 3, H and I), suggesting the disintegrative role of Angpt 2 in the microvasculature of the ischemic heart. 
A
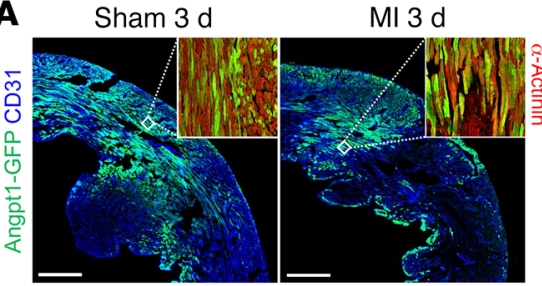

B

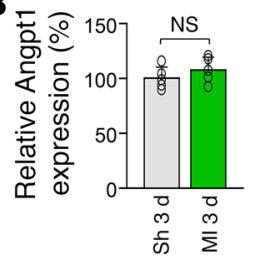

C

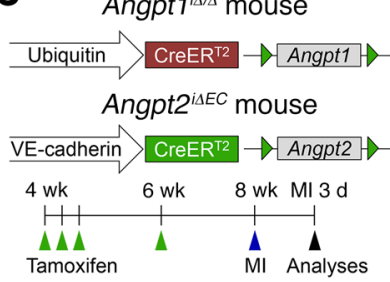

D

Sham $3 d$
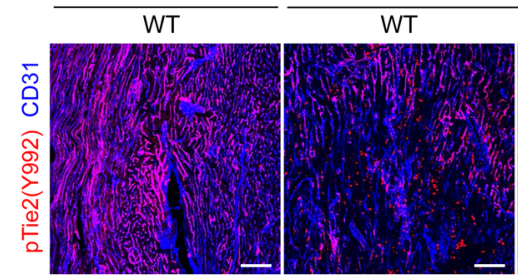

MI 3 d

Angpt $1^{1 / 4}$
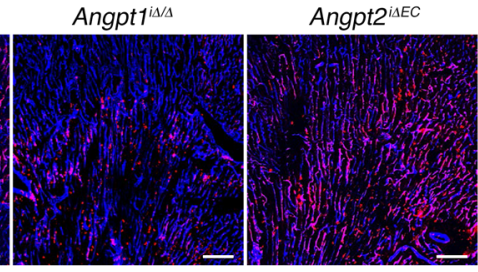

E

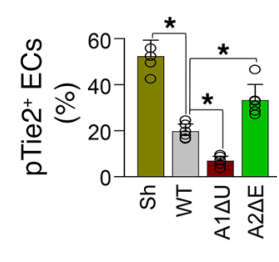

F

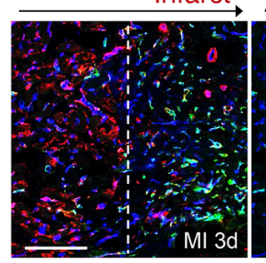

Angpt2 NG2 CD31

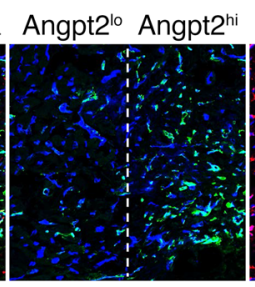

Angpt2 CD31

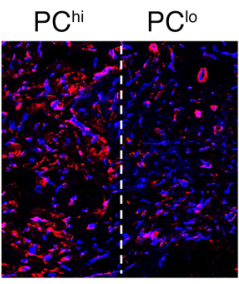

NG2 CD31

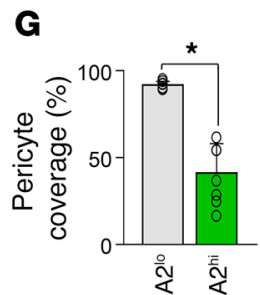

H

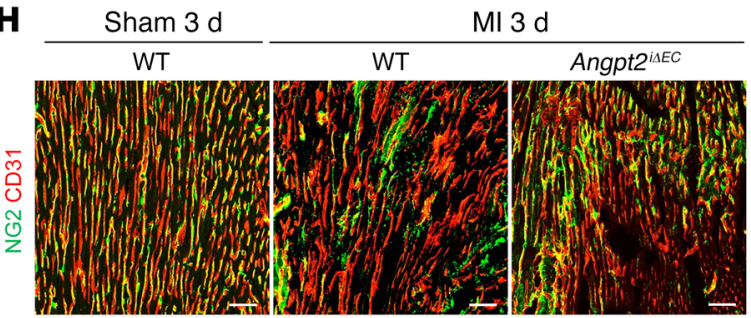

I
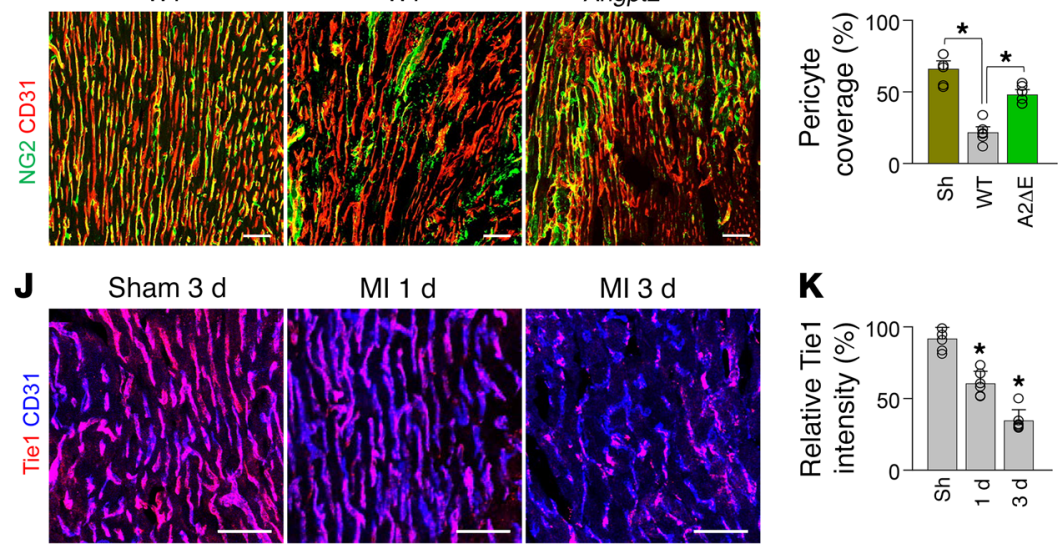

K

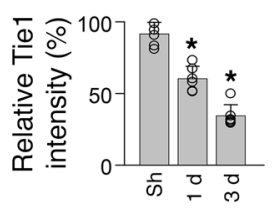

Figure 3. Angpt2 destabilizes endothelial integrity of ischemic heart by antagonizing Angpt1/Tie2 signal-

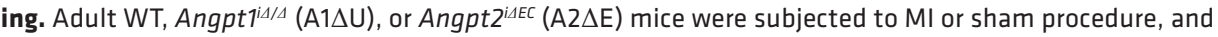
hearts were harvested at 3 days after MI. (A and B) Images and comparisons of Angpt1 expression in heart of Angpt1-GFP mice at 3 days after sham or MI procedure. Each region marked by a box demonstrating the expression of Angpt1 in myocardium is magnified in the right corner. Scale bars: $500 \mu \mathrm{m} . n=4-5$. (C) Diagram depicting generation of Angpt $7^{i / \Delta}$ or Angpt $2^{i \Delta E C}$ mice and experiment schedule. (D and E) Immunofluorescence images and quantification show the decrease in pTie2 in border zone ECs after Ml; pTie2 level was lower in Angpt $7^{i / 4}$ mice, but higher in Angpt2 $2^{\text {iLEC }}$ mice compared with WT. Scale bars: $100 \mu \mathrm{m} . n=5-6$, each time point. ${ }^{*} P<0.01$, Kruskal-Wallis test followed by Mann-Whitney $U$ test for post hoc pairwise comparisons. (F and $\mathbf{G}$ ) Images and comparisons of $\mathrm{NG2}^{+}$pericyte $(\mathrm{PC})$ coverage on $\mathrm{ECs}$ at the border zone. Direction of arrow indicates the border zone closer to infarct area. Dashed lines distinguish PChi/Angpt $2^{10}$ region from $\mathrm{PC}^{\mathrm{lo}} /$ Angpt $^{\mathrm{hi}}$ region at the infarct border. $\mathrm{PC}$ coverage was compared between the ECs within high Angpt2 expression zone $\left(A 2^{\mathrm{hi}}\right)$ and low Angpt2 expression zone $\left(A 2^{10}\right) . n=4$, each group. Scale bars: $100 \mu \mathrm{m} .{ }^{*} P<$ 0.05 versus $A 2^{10}$ zone, Mann-Whitney $U$ test. ( $H$ and $\mathbf{I}$ ) Images and comparisons of $N G 2^{+} P C$ coverage onto ECs at the border zone. $n=5-6$, each group. Scale bars: $50 \mu \mathrm{m}$. ${ }^{*} P<0.025$, Kruskal-Wallis test followed by Mann-Whitney $U$ test for post hoc pairwise comparisons (WT versus sham and WT versus Angpt $2^{i \Delta E C}$ ). (J and K) Temporal changes of relative expression of Tie 1 in the ECs of infarct border. $n=5-6$, each time point. Scale bars: $50 \mu \mathrm{m} .{ }^{*} P<0.05$ versus sham, Mann-Whitney $U$ test. Error bars represent mean \pm SD.
Tie1 is an endothelial orphan receptor and a homolog of Tie2 that positively modulates Tie2 signaling $(33,34)$. Given that Tie1 expression is reduced during inflammation and that this reduced Tie1 allows enhanced antagonistic action of Angpt2 against Tie2 $(25,34,35)$, we were curious about the expression level of the Tie1 protein in ECs of the border zone. Immunohistological analysis revealed that Tie1 was highly present in cardiac ECs of sham-operated control mice, but marked and gradual decline of Tie1 was detected at days 1 and 3 after MI (Figure 3, J and K). This finding implies that the antagonistic action of Angpt2 to Tie2 could be enforced in the ECs at the border zone. To further confirm the observed changes in immunofluorescence staining, expression of each protein after MI was investigated by immunoblotting or the immunoprecipitation method, which showed consistent results with immunostaining (Supplemental Figure 4, A-D).

EC-derived Angpt2 exacerbates vascular leakage, cardiac hypox$i a$, and infarction. We and others recently reported that Angpt2 mediates vascular leakage in severe inflammatory conditions, leading to impairment of microvascular perfusion and organ damage $(23,36)$. To investigate whether EC-derived Angpt2 and its Tie2-antagonizing action on ECs at the border zone contribute to vascular disintegration and infarct progression, Angpt $2^{\text {iAEC }}$ (gene deletion efficiency, 93\%; Supplemental Figure 5 and Supplemental Table 1) and WT mice were subjected to the MI procedure or to a sham procedure, and vascular leakage, perfusion, hypoxic molecule expression, and the infarct size were compared 3 days after surgery. Vascular leakage of low-molecular weight $(40 \mathrm{kDa})$ dextran from the border zone microvasculature was markedly reduced, by $71 \%$, 
A

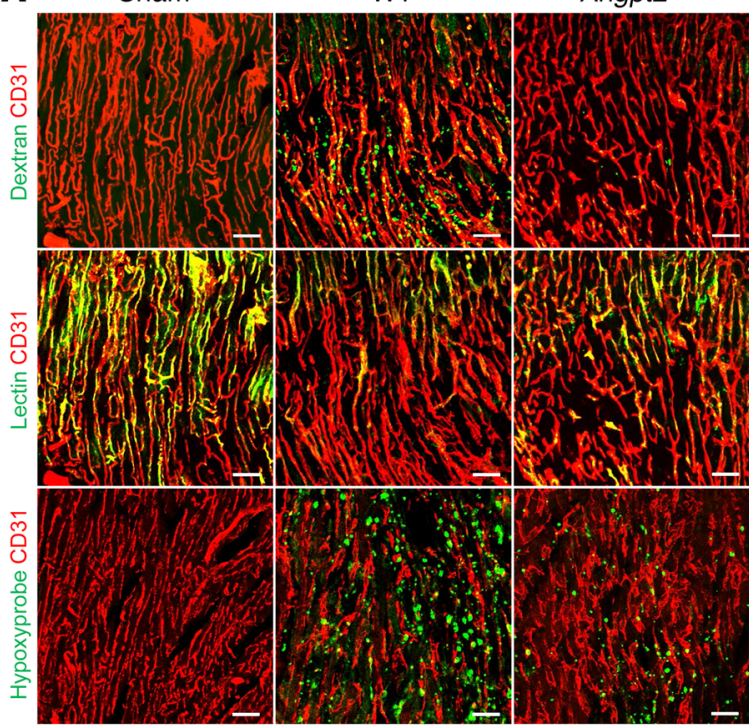

C Sham

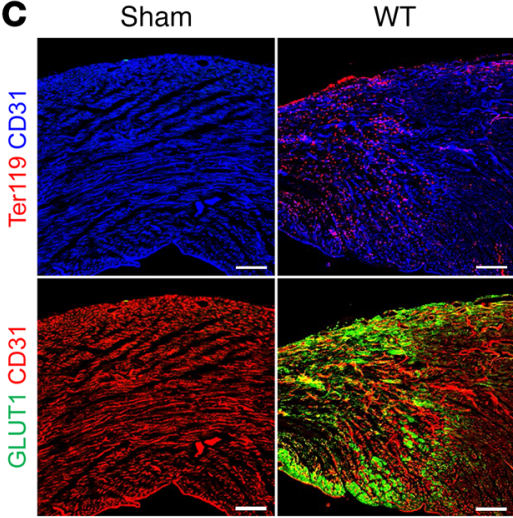

E

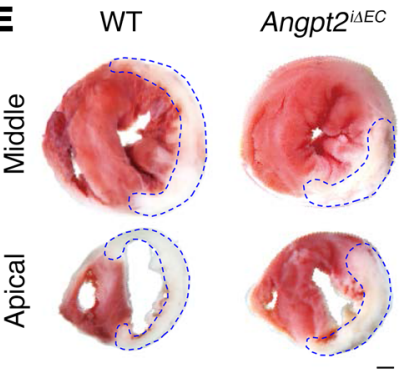

B
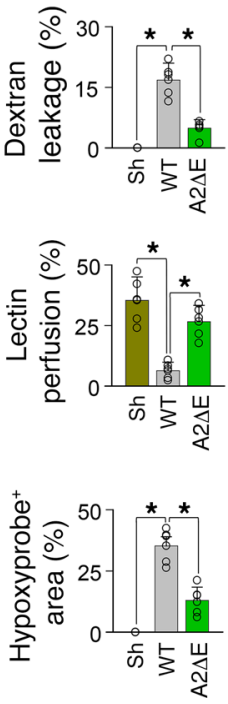

Angpt $1^{i / \Delta}$

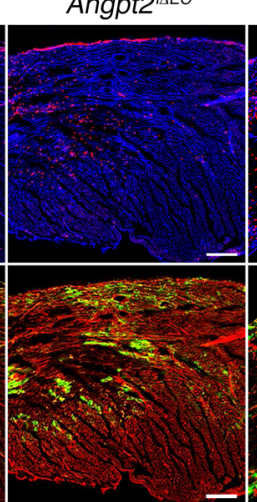

$\mathbf{F}$

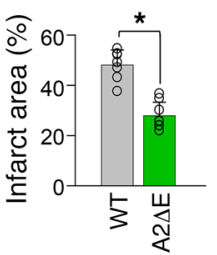

Figure 4. EC-specific depletion of Angpt2 mitigates vascular leakage and improves microvascular perfusion and tissue oxygenation in ischemic heart. Adult WT, Angpt $7^{i / / 4}$ or Angpt2 $2^{\text {iLEC }}$ mice were subject to $\mathrm{Ml}$ or sham procedure, and hearts were harvested at 3 days after MI. (A and B) Images and comparisons of dextran leakage, FITC-lectin perfusion, and Hypoxyprobe $^{+}$hypoxic area. $n=5-6$, each group. Scale bars: $50 \mu \mathrm{m} .{ }^{*} P<$ 0.025 , Kruskal-Wallis test followed by Mann-Whitney $U$ test for post hoc pairwise comparisons. (C and D) Images and comparisons of TER119+ RBC leakage, and GLUT1+ hypoxic area. $n=5-6$, each group. Scale bars: 200 $\mu \mathrm{m} .{ }^{*} P<0.01$, Kruskal-Wallis test followed by Mann-Whitney $U$ test for post hoc pairwise comparisons. (E and $\mathbf{F}$ ) Representative images of TTC-stained cross-sections at middle and apical portions of hearts. White area demarcated by dashed lines corresponds to infarcted region. Each infarcted area per total area in the middle section is compared. $n=7$, each group. Scale bar: $500 \mu \mathrm{m} .{ }^{*} P<0.05$ versus WT, Mann-Whitney $U$ test. Error bars represent mean $\pm \mathrm{SD}$. in Angpt $2^{i \Delta E C}$ mice, and lectin perfusion was improved by $69 \%$ in Angpt ${ }^{i \Delta E C}$ mice compared with WT mice (Figure 4, A and B). Consequently, cardiac hypoxia as measured by Hypoxyprobe- 1 staining was improved by $63 \%$ in Angpt $2^{\mathrm{i} \triangle \mathrm{EC}}$ mice compared with WT mice (Figure 4, A and B). To gain further insight into the contributing role of the Angpt-Tie2 system on vascular integrity after MI, Angpt $1^{i / / 4}$ and Tie $2^{i \Delta E C}$ mice were also subjected to MI or sham procedure, and the impact of the deletion of either gene on vascular leakage and hypoxia was investigated. We generated Tie $2^{i \Delta E C}$ mice by crossing Tie $2^{f / f l}$ mice (37) with VE-cadherin-Cre-ER ${ }^{\mathrm{T} 2}$ mice (28) (Supplemental Figure 6A) and depleted Tie2 in a tamoxifeninducible manner (deletion efficiency, 94\%; Supplemental Figure 5 and Supplemental Table 1). Leakage of RBCs and expression of GLUT-1, another marker for hypoxia (38), were signifi- cantly mitigated in Angpt $2^{i \triangle E C}$ mice compared with WT mice. On the other hand, leakage and hypoxia were further exacerbated in Angpt $1^{i / \Delta}$ mice (Figure 4, C and D) and Tie $2^{i \Delta E C}$ mice (Supplemental Figure 6, B and C) compared with WT mice. Indeed, the sizes of infarcts in Angpt $2^{i \Delta E C}$ mice were significantly smaller (42\%, $P=$ 0.008 ) than those in WT mice (Figure 4, E and F). Together, these results indicate that, after MI, EC-derived Angpt2 could antagonize endothelial Tie2 phosphorylation and exacerbate cardiac hypoxia and infarct progression by aggravating vascular leakage and microvascular hypoperfusion.

EC-derived Angpt2 aggravates vascular inflammation and myocardial remodeling after MI. An excessive and uncontrolled inflammatory response after MI exacerbates the pathological remodeling of the infarcted cardiac tissue (39). Given the augmenting role 
A
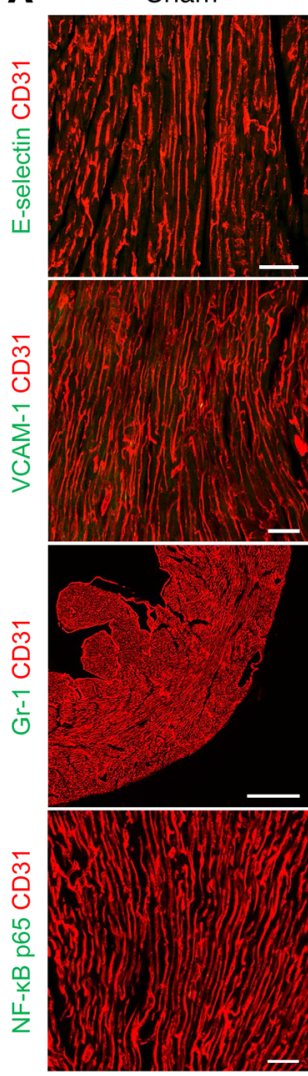

WT

C

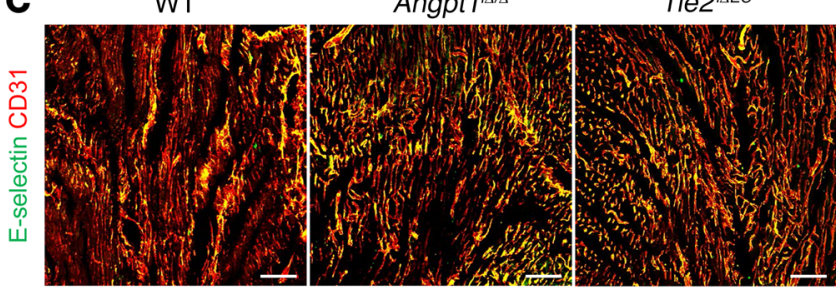

WT
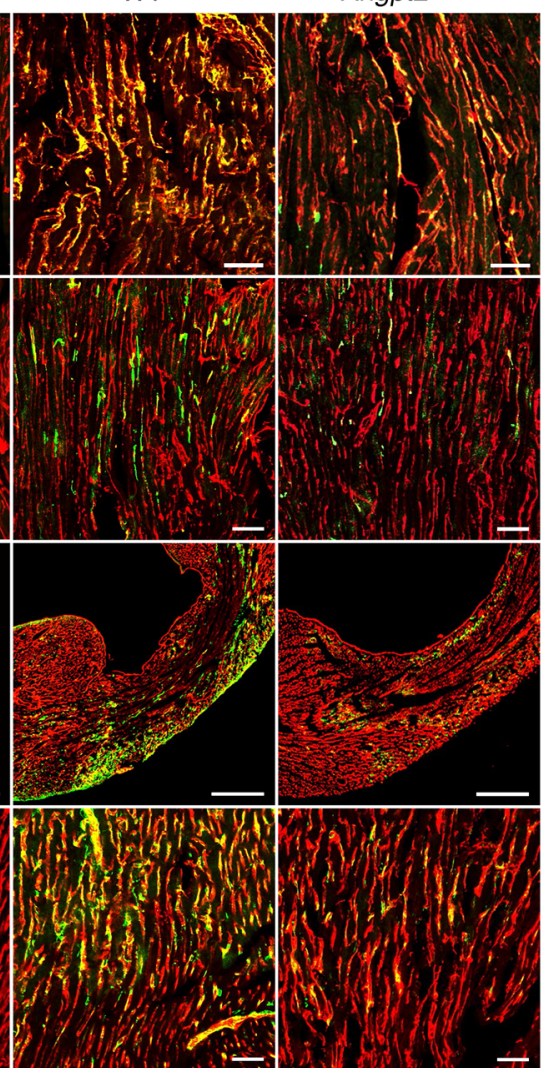

Tie2 $2^{i \Delta E C}$

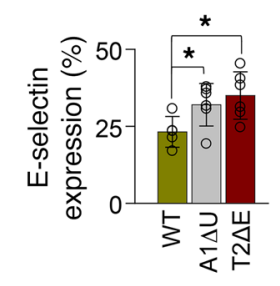

B
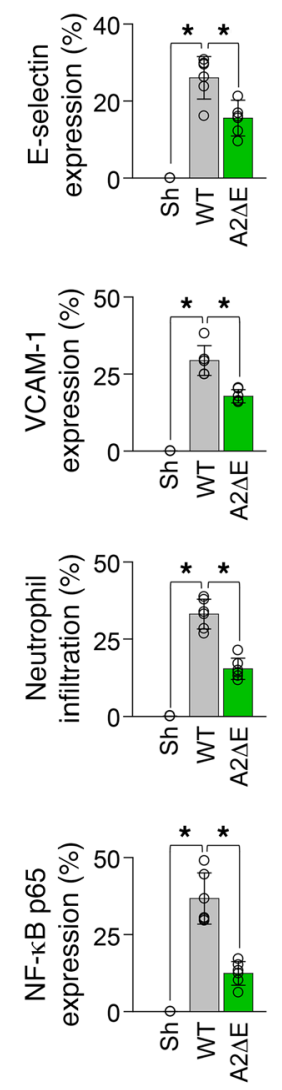

D
Figure 5. EC-specific depletion of Angpt2 ameliorates expressions of adhesion molecules and neutrophil infiltration in ischemic heart. Adult WT, Angpt $7^{i / 4}$, Angpt $^{i \Delta E C}$, or Tiez ${ }^{i \Delta E C}(\mathrm{~T} 2 \Delta \mathrm{E})$ mice were subject to $\mathrm{MI}$ or sham procedure, hearts were harvested at 3 days after $\mathrm{Ml}$, and indicated molecules in heart sections at the infarct border were detected by immunostaining. (A and $\mathbf{B}$ ) Images and comparisons of E-selectin, VCAM-1, and NF- $\kappa B$ p65 on ECs and $\mathrm{Gr}-1^{+}$neutrophil infiltration in the infarct border. $n=5-6$, each group. Scale bars: 50 $\mu \mathrm{m}$, except for $\mathrm{Gr}-1^{+}$neutrophil infiltration (500 $\mu \mathrm{m})$. (C and $\mathbf{D})$ Images and comparisons of E-selectin expression on ECs at the infarct border. $n=5-6$, each group. Scale bars: $100 \mu \mathrm{m} .{ }^{*} P<0.025$, Kruskal-Wallis test followed by Mann-Whitney $U$ test for post hoc pairwise comparisons. Error bars represent mean \pm SD.

Heparan sulfate (HS) is a major component of the endothelial glycocalyx (eGC) and cardiac ECM (cECM). HS is degraded by heparanase, which is upregulated and activated by NF- $\mathrm{\kappa B}$ activation during inflammation (40, 41), and suppressed by Tie 2 activation (23). Therefore, we asked whether ECderived Angpt2 and subsequent upregulation of NF- $\mathrm{kB}$ p65 expression and perturbation of Tie2 activity play roles in eGC and cECM degradation by enhancing heparanase activity in ECs after MI. Indeed, heparanase expression was markedly increased in ECs at the border zone and in the surrounding infiltrated CD $45^{+}$leukocytes 3 days after MI, while no heparanase was detected in the hearts of sham-operated control mice (Figure 6, A and B). Immunoblot analyses also demonstrated marked upregulation of both inactive and active heparanase at the infarct border after MI (Figure 6, C and D). Using Image software (NIH), we defined each $\mathrm{HS}$ of eGC or cECM, termed HS-eGC and HS-cECM, respectively (Supplemental Figure 8, A and B). Well-aligned distributions of HS-eGC and HS-cECM were detected in hearts of normal and sham-operated control mice (Figure 6E and Supplemental Figure 8A). Compared with sham-operated control heart, severe losses and disorganizations of total HS, HS-eGC, and HS-cECM were detected in the ischemic regions of hearts 3 days after MI (Figure 6, E and F, Supplemental Figure 8B). However, compared with WT MI mice, Angpt $2^{\mathrm{i} E \mathrm{C} C} \mathrm{MI}$ mice showed reduced heparanase levels $(-57 \%)$ and preserved total HS $(\sim 52 \%)$, HS-eGC ( $\sim 59 \%)$, and HS-cECM $(\sim 26 \%)$ in the ischemic region 3 days after MI (Figure 6, E and F), suggesting a close correlation between the Angpt2-mediated heparanase expression and HS degradation on the EGC and ECM. On the other hand, expression of heparanase in ECs at the border zone and infiltrated leukocytes were further exaggerated in Angpt $1^{\mathrm{i} / /}$ mice and Tie ${ }^{\text {iLEC }}$ mice compared with WT mice (Supplemental Figure 9, A and B), implying the suppressive role of endothelial Tie2 on heparanase expression in ischemic heart. Together, these results prevents vascular inflammation. 

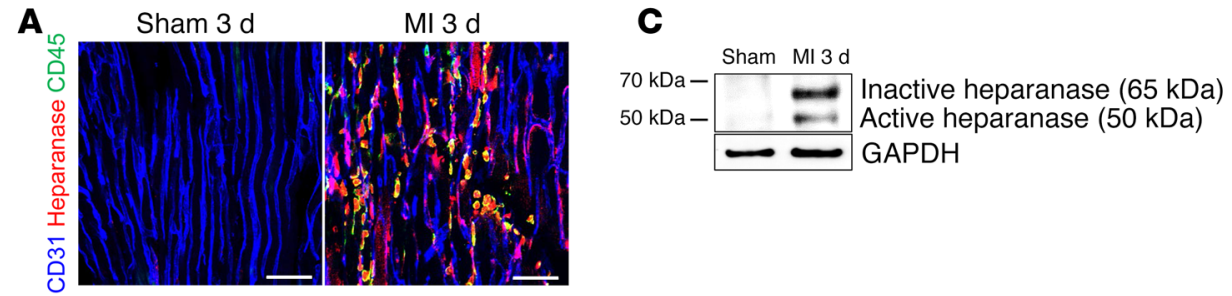

$\mathbf{B}$
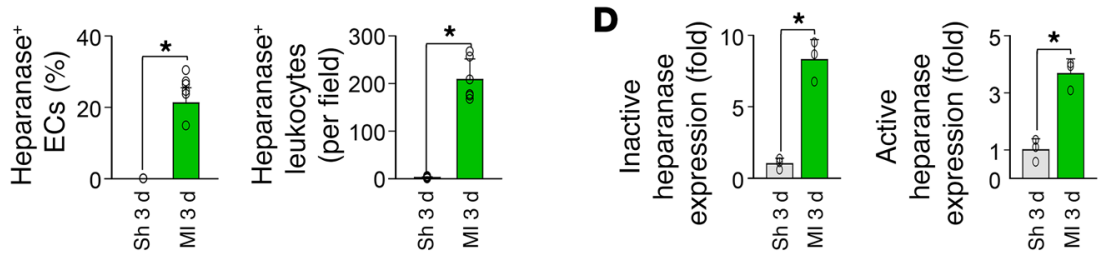

\section{$\mathbf{E}$}
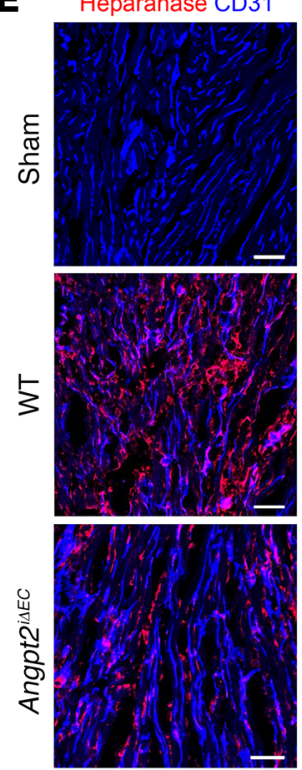

HS CD31
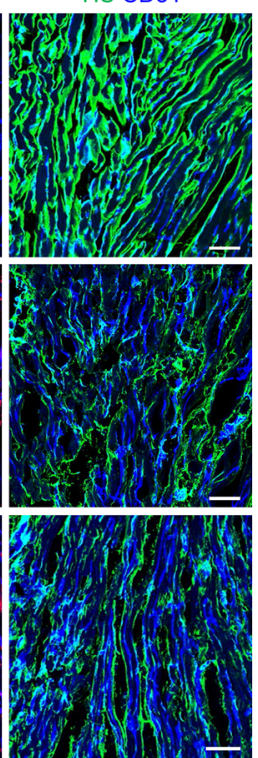
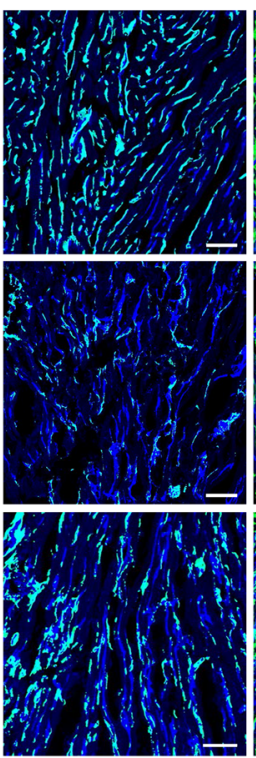
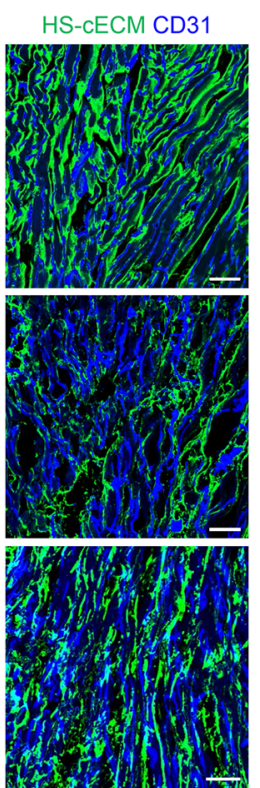
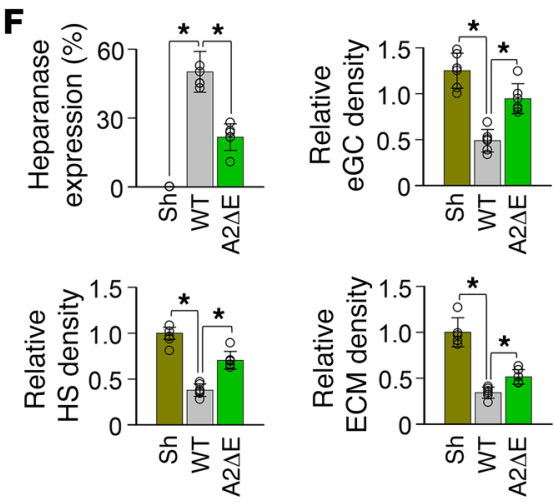

Figure 6. EC-specific depletion of Angpt2 mitigates degradation of eGC and ECM in ischemic heart. Adult WT or Angpt2iAEC mice were subject to MI or sham procedure, hearts were harvested at 3 days after MI, and indicated molecules in heart sections at the infarct border were detected by immunostaining. (A and B) Images and comparisons showing increased heparanase in ECs and infiltrating CD45+ leukocytes. $n=5-6$, each group. Scale bars: $50 \mu m$. ${ }^{*} P<0.05$ versus sham, Mann-Whitney $U$ test. (C and $\mathbf{D}$ ) Immunoblot and densitometric analyses of heparanase expression and activation at the infarct border after MI. $n=3$, each group. ${ }^{*} P<0.05$ versus sham, Mann-Whitney $U$ test. (E and F) Images and comparisons of heparanase expression, HS-eGC density, and HS-cECM density. $n=5-6$, each group. Scale bars: $50 \mu \mathrm{m}$. ${ }^{*} P<0.025$, Kruskal-Wallis test followed by Mann-Whitney $U$ test for post hoc pairwise comparisons. Error bars represent mean $\pm \mathrm{SD}$.

indicate that Angpt2 augments vascular destabilization, vascular inflammation, and adverse cardiac remodeling that is mediated by antagonizing Tie2 signaling during the acute phase of MI.

Angpt2 plays a facilitating role in macrophage polarization toward a proinflammatory type. Macrophages have considerable plasticity to adopt phenotypical and functional alterations, called macrophage polarization, in response to inflammatory stimuli (42). In particular, proinflammatory M1-like macrophages promote tissue damage and adverse cardiac remodeling after MI (43). In contrast, those with antiinflammatory properties, termed M2-like macrophages, mitigate myocardial injury (44). Histological analysis of the infarct border in MI mice revealed unexpected but distinct Angpt2 expression in $\mathrm{CD} 68^{+}$macrophages 7 days after MI (Figure 7, A and B). This is in accordance with previous in vitro findings (45) that hypoxia-induced HIF-1 $\alpha$ promotes Angpt2 expression by macrophages. Notably, approximately $93 \%$ of Angpt $2^{+}$macrophages coexpressed inducible NOS (iNOS), a proinflammatory marker (Figure 7A). We therefore questioned whether Angpt2 plays a substantial role in macrophage polarization. However, the infiltrated macrophages hardly expressed Tie2 (Figure 7C). On the other hand, Angpt2 is known to induce the phosphorylation of ERK- and FAK-signaling pathways by binding to integrins $\alpha_{v} \beta_{3}, \alpha_{v} \beta_{5}$, and $\alpha_{5} \beta_{1}$ in Tie2-low or Tie2-negative cells (11), and ERK activation induces the proinflammatory polarization of macrophages (46). Intriguingly, CD $68^{+}$cardiac macrophages highly expressed integrin $\alpha_{5} \beta_{1}$ (Figure 7, C and D), most of which colocalized with Angpt2 (Figure 7E). Therefore, we reasoned that Angpt2 might play an important role in polarizing macrophages toward a proinflammatory state by bind- 
A

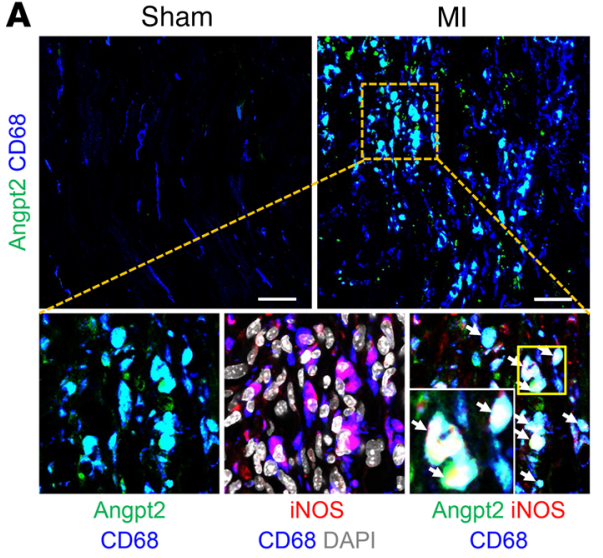

B
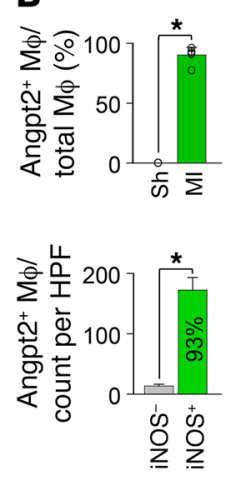

C

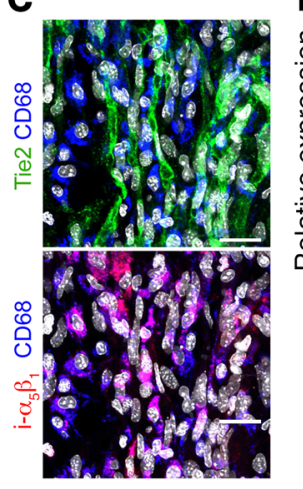

$\mathbf{E}$

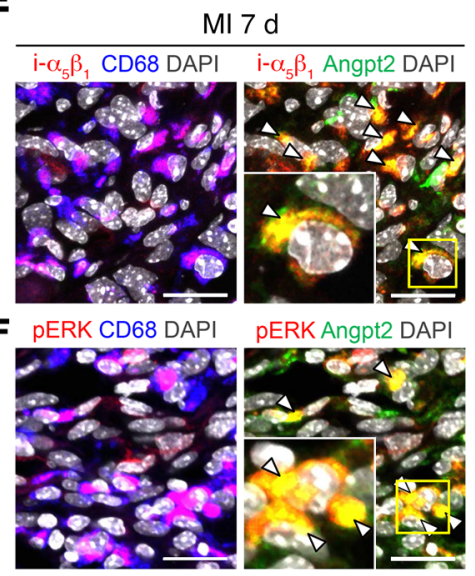

G

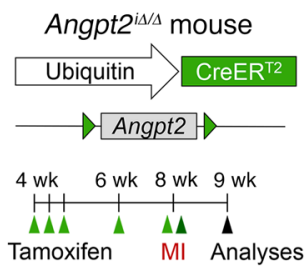

H

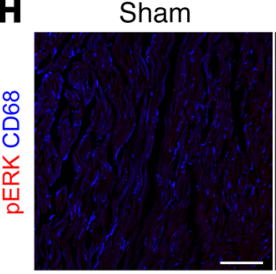

WT

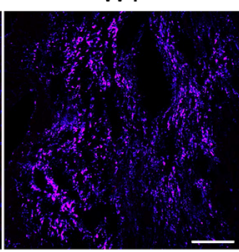

Figure 7. Angpt2/integrin $\alpha_{5} \beta$ signaling is positively associated with pERK expression in macrophages in ischemic heart. Adult WT or Angpt $2^{i / / \Delta}(\mathrm{A} 2 \Delta \mathrm{U})$ mice were subject to $\mathrm{Ml}$ or sham procedure, hearts were harvested at 7 days after $\mathrm{Ml}$, and indicated molecules in heart sections at the border zone were detected by immunostaining. (A and $\mathbf{B}$ ) Images and comparisons of Angpt2 expression in $\mathrm{CD}_{6} 8^{+}$macrophages. Box region is magnified below. Note colocalization of Angpt2 and iNOS (white arrows). $n=5-6$, each group. Scale bars: $50 \mu \mathrm{m}$. HPF, high-power field. ${ }^{*} P<0.05$ by Mann-Whitney $U$ test. ( $\mathbf{C}$ and $\mathbf{D}$ ) Images and comparisons of expression of Tie2 and integrin $\alpha_{5} \beta_{1}\left(i-\alpha_{5} \beta_{1}\right.$ ) in $\mathrm{CD}^{+} 8^{+}$macrophages. Scale bars: $20 \mu \mathrm{m} . n=4-5$, each group. ${ }^{*} P<0.05$ by Mann-Whitney $U$ test. (E) Images showing colocalization of integrin $\alpha_{5} \beta_{1}$ and Angpt2 in CD68 ${ }^{+}$macrophages (white arrowheads). Scale bars: $20 \mu \mathrm{m}$. (F) Images showing colocalization of pERK (at Thr202/Tyr 204) and Angpt2 in CD68+ macrophages (white arrowheads). Scale bars: $20 \mu \mathrm{m}$. (A, E, and F) Each area marked by a yellow box is magnified in the left bottom corner. (G) Diagram depicting generation of Angpt $2^{i / 4}$ mice and their experiment schedule. ( $\mathbf{H}$ and $\mathbf{I}$ ) Images and comparisons of pERK in CD68+ macrophages. $n=5-6$, each group. Scale bars: $100 \mu \mathrm{m}$. ${ }^{*} P<0.025$, Kruskal-Wallis test followed by Mann-Whitney $U$ test for post hoc pairwise comparisons. (J) Diagram depicting the experiment schedule for integrin $\alpha_{5} \beta_{1}$ inhibitor treatment (ATN-161, $30 \mathrm{mg} / \mathrm{kg}$, intraperitoneal injection) after MI. (K and $\mathbf{L}$ ) Images and comparisons of pERK in CD68 ${ }^{+}$macrophages. $n=4-5$, each group. Scale bars: $100 \mu \mathrm{m}$. ${ }^{*} P<0.05$, Mann-Whitney $U$ test. Error bars represent mean $\pm \mathrm{SD}$.

ing to the integrin $\alpha_{5} \beta_{1}$ receptor and activating ERK. Indeed, ERK phosphorylation, as determined by phosphospecific antibody recognition of ERK1/2 (Thr202/Tyr204), was observed in most of the Angpt $2^{+}$macrophages ( $95 \%$ ) (Figure $7 \mathrm{~F}$ ). Therefore, we supposed that the upregulated Angpt2 expression in macrophages and ECs
D

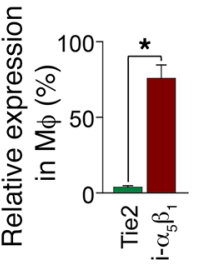

I

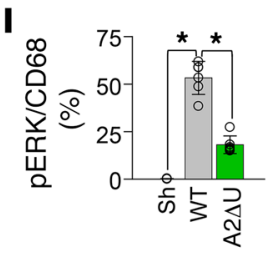

Angpt2 $^{i \Delta / \Delta}$

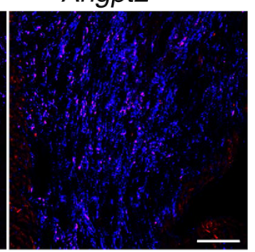

$\mathbf{L}$

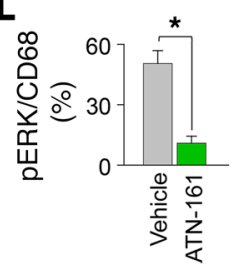

may induce proinflammatory macrophage polarization that is mediated by the activation of the integrin $\alpha_{5} \beta_{1} /$ ERK-signaling pathway in autocrine and paracrine manners.

To determine whether Angpt2 plays a facilitating role in proinflammatory macrophage polarization through the integrin $\alpha_{5} \beta_{1} /$ ERK-signaling pathway, we used globally Angpt2-depleted (Angpt2i//4) mice that were generated by crossing Angpt $2^{f / f l}$ mice (47) with UBC-Cre-ER ${ }^{\mathrm{T} 2}$ mice (Figure 7G). Compared with WT mice, in Angpt $2^{i / 4}$ mice (gene deletion efficiency, 85\%, Supplemental Figure 5 and Supplemental Table 1), phosphorylated ERK (pERK) expression in $\mathrm{CD}^{+} 8^{+}$macrophages was reduced by $66 \%$ at 7 days after MI (Figure 7, $\mathrm{H}$ and I). We also determined whether inhibition of integrin $\alpha_{5} \beta_{1}$ mitigates pERK expression in $\mathrm{CD}^{+} 8^{+}$ cardiac macrophages. Treatment of ATN-161, a specific peptide-based inhibitor against integrin $\alpha_{5} \beta_{1}$, after MI (Figure 7J) also markedly reduced pERK expression levels in $\mathrm{CD}^{+} 8^{+}$ macrophages, by $78 \%$ (Figure $7, \mathrm{~K}$ and $\mathrm{L}$ ), supporting our hypothesis that Angpt2/integrin $\alpha_{5} \beta_{1}$ signaling contributes to ERK phosphorylation of $\mathrm{CD} 68^{+}$ macrophages after MI. Further, we evaluated the expression profile of pro- and antiinflammatory markers in $\mathrm{CD} 68^{+}$macrophages of WT and Angpt2 $2^{i / 4}$ mice at 7 days after MI (Figure 8A). Expression of iNOS and MHC II in CD68 $8^{+}$acrophages was reduced in Angpt $2^{i / / 4}$ mice by approximately $57 \%$ and approximately $56 \%$, respectively, while the expression of CD206 and arginase-1 (Arg-1), which are antiinflammatory markers, was not significantly different, resulting in a decreased iNOS/CD206 ratio (Figure 8, B and C). Treatment of integrin $\alpha_{5} \beta_{1}$-inhibitory peptide also remarkably mitigated iNOS expression in CD68 $8^{+}$macrophages, by 69\% (Figure 8, D-F). Taken together, these findings suggest that Angpt2/ 
A Angpt2is/s mouse

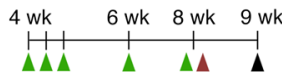

Tamoxifen MI Analyses

B inOS CD206 CD68
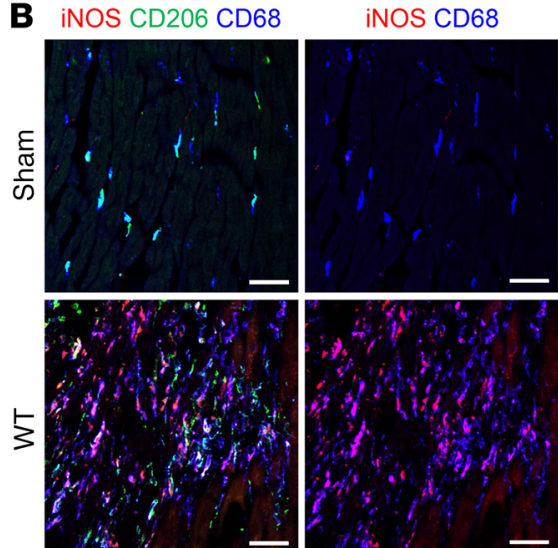

CD206 CD68
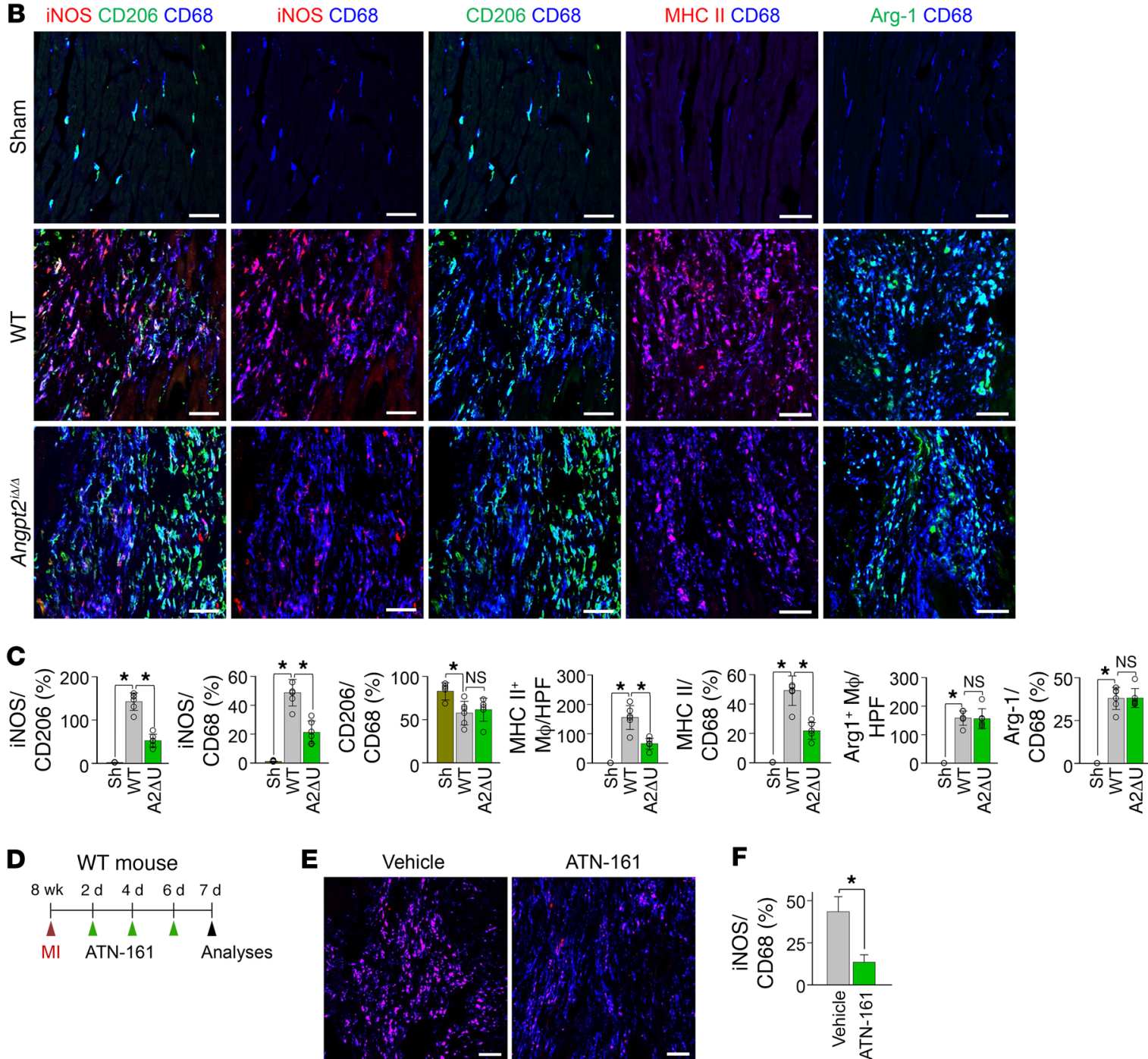

ATN-161
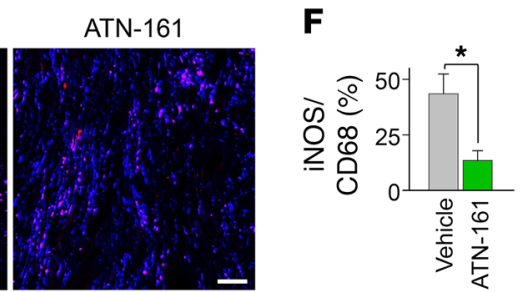

iNOS CD68

Figure 8. Angpt2 plays a substantial role in proinflammatory macrophage polarization in ischemic heart. Adult WT or Angpt $2^{i / /}$ mice were subject to MI or sham procedure, hearts were harvested at 7 days after $\mathrm{MI}$, and indicated molecules in heart sections at the infarct border were detected by immunostaining. (A) Diagram depicting generation of Angpt $2^{i / 4}$ mice and their experiment schedule. (B) Images of expression of iNOS, CD206, MHC II, and Arg-1 in CD68 ${ }^{+}$macrophages. Scale bars: $50 \mu \mathrm{m}$. (C) Comparisons of indicated parameters. $n=5-6$, each group. ${ }^{*} P<0.025$. Kruskal-Wallis test followed by Mann-Whitney $U$ test for post hoc pairwise comparisons. Significance was adjusted for multiple comparisons using Bonferroni's method. Error bars represent mean \pm SD. (D) Diagram depicting the experiment schedule for integrin $\alpha_{5} \beta_{1}$ inhibitor treatment (ATN-161, $30 \mathrm{mg} / \mathrm{kg}$, intraperitoneal injection) after MI. (E and F) Images and comparisons of iNOS expression in CD68 ${ }^{+}$macrophages. $n=4-5$, each group. Scale bars: $100 \mu \mathrm{m}$. ${ }^{*} P<0.05$, Mann-Whitney $U$ test. Error bars represent mean $\pm \mathrm{SD}$.

integrin $\alpha_{5} \beta_{1}$ signaling plays an important role in establishing an inflammatory microenvironment after myocardial ischemia by promoting proinflammatory macrophage polarization.

To elucidate whether macrophage- or endothelial-driven Angpt2 plays a major role in macrophage polarization, we generated Angpt $2^{\Delta M \Phi}$ mice by crossing Angpt $2^{f / f l}$ mice (27) with LysM-Cre mice (48) (Supplemental Figure 10, A-C). Macrophage-specific depletion of Angpt 2 markedly diminished ERK phosphorylation and proinflammatory macrophage polarization, while EC-specific depletion exerted negligible effects (Supplemental Figure 10, D-G), implying the dominant role of macrophage-derived Angpt2 in determination of macrophage polarity after MI.

Angpt2 continuously drives adverse vascular remodeling in the infarct border zone. We further investigated whether Angpt2 has a sustained effect on vascular remodeling by analyzing the infarct border zone 2 and 3 weeks after MI. Of special note, Angpt 2 expression was still high in the ECs at these time points (Figure 9, A and B). Notably, severely disorganized or disintegrated vessels at the border 
A Sham 2 wk

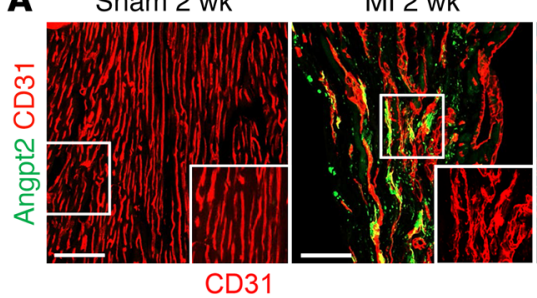

D

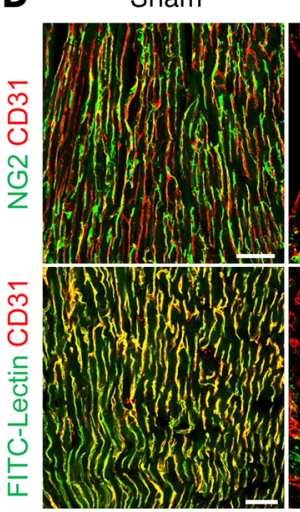

$\mathbf{F}$
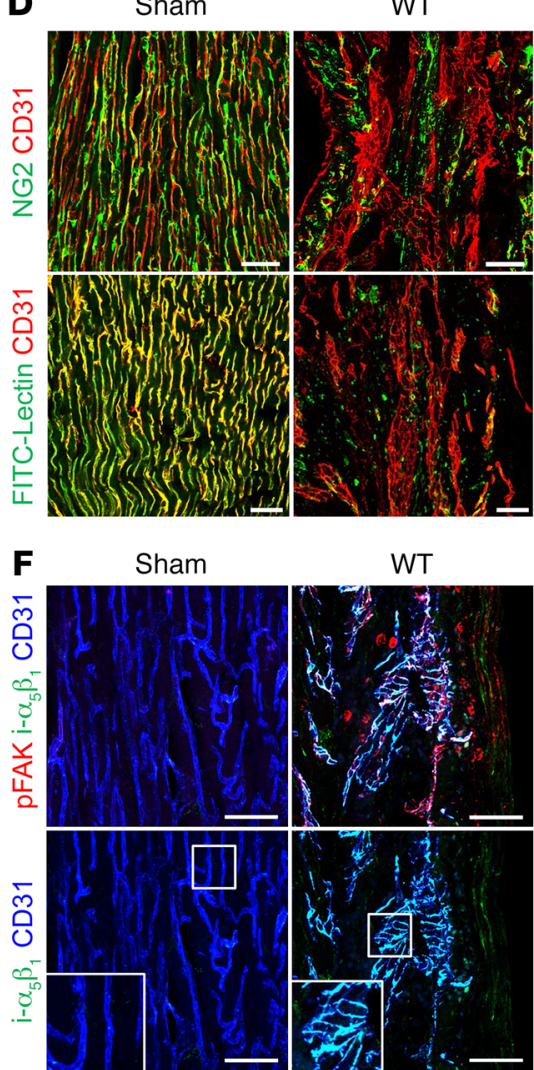

Angpt2 $^{i / / 4}$

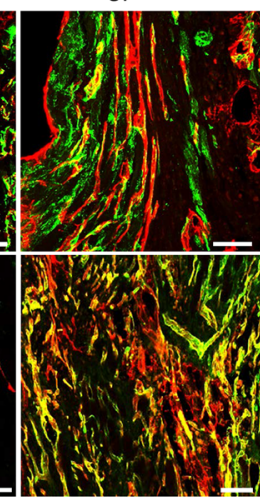

E

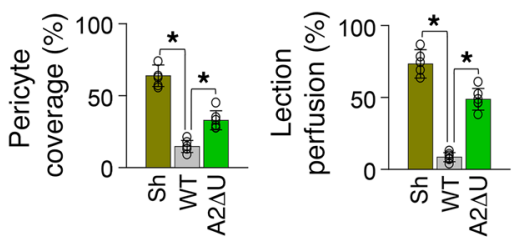

B

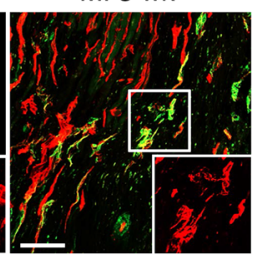

C

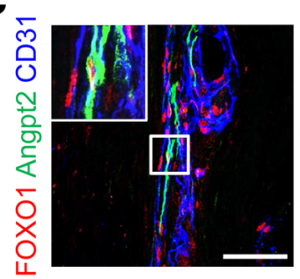

Figure 9. Angpt2 contributes to adverse vascular remodeling, thereby inhibiting effective microvascular perfusion in chronically ischemic heart. Adult WT or Angpt $2^{i / / 4}$ mice were subject to MI or sham procedure, hearts were harvested at 2 weeks after MI, and indicated molecules in heart sections at the infarct border were detected by immunostaining. (A and $\mathbf{B}$ ) Images and comparisons of Angpt2 in ECs of remodeling vessels at the border zone. Each box region is magnified in right corner. Note high Angpt2 in the disintegrated $\mathrm{ECs} . n=5$, each group. Scale bars: $50 \mu \mathrm{m}$. (C) Images showing nuclear localization of FOXO1 in Angpt2 ${ }^{+} \mathrm{ECs}$ of the remodeling vessels. Boxed region is magnified in left corner. Scale bar: $50 \mu \mathrm{m}$. (D and $\mathbf{E}$ ) Images and comparisons of $\mathrm{NG2}^{+}$pericyte coverage and FITC-lectin perfusion in ECs. $n=$ 5-6, each group. Scale bars: $50 \mu \mathrm{m}$ ( $\mathbf{F}$ and $\mathbf{G}$ ) Images and comparisons of $\mathrm{i}-\alpha_{5} \beta_{1}$ and $\mathrm{FAK}$ phosphorylation at $\mathrm{Tyr}^{397}$ (pFAK-Tyr 397) in ECs.

Each boxed region is magnified in left corner. $n=5-6$, each group. Scale bars: $50 \mu \mathrm{m}$. (H) Immunoblot images showing reduced Angpt2-induced FAK phosphorylation in HUVECs transfected with siRNAs for integrin $\alpha_{5}$ (silTCA5), integrin $\beta_{1}$ (silTCB1), or scrambled control (siCont). (B, E, and G) Comparisons of indicated parameters. ${ }^{*} P<0.025$, Kruskal-Wallis test followed by Mann-Whitney $U$ test for post hoc pairwise comparisons. Significance was adjusted for multiple comparisons using Bonferroni's method. Error bars represent mean $\pm \mathrm{SD}$.

zone exhibited higher Angpt2 levels and distinct FOXO1 nuclear localization (Figure 9, A-C), indicating that disrupted ECs retained activated FOXO1 transcriptional activity and thereby increased Angpt2 expression in a positive feedback manner. Compared with sham-operated control hearts, marked impairments of $\mathrm{NG}^{+}$pericyte coverage, collagen type $\mathrm{IV}^{+}(\mathrm{Col} \mathrm{IV})$ basement membrane, and $\mathrm{ZO}-1^{+}$tight junction on the ECs were detected at the infarct border zone 2 weeks after MI. To investigate whether Angpt2 from any source, i.e., from either ECs or from polarized macrophages, plays a role in chronic vascular remodeling, we used Angpt $2^{i \Delta / \Delta}$ mice rather than Angpt $2^{i \Delta E C}$ mice. Compared with those from WT mice, the ECs of Angpt $2^{i / \Delta}$ mice showed better $\mathrm{NG}^{+}$pericyte coverage ( $\left.37 \%\right)$, more $\mathrm{Col} \mathrm{IV} \mathrm{V}^{+}$basement membrane $(\sim 40 \%)$, more $\mathrm{ZO}-1^{+}$tight junctions $(\sim 47 \%)$, and greater FITC-lectin ${ }^{+}$perfusion ( $\left.62 \%\right)$ (Figure 9, D and E, and Supplemental Figure 11, A and B).
To gain insights into the mechanisms involved in Angpt2induced vascular remodeling, we examined the expression of putative receptors and signaling molecules downstream of Angpt2. Of note, strong expression of integrin $\alpha_{5} \beta_{1}$, which is not expressed in ECs of sham-operated control hearts, was observed in ECs at border zones of chronic ischemic hearts of WT and Angpt $2^{i \Delta / \Delta}$ mice (Figure 9, F and G). FAK phosphorylation, as detected by phosphospecific antibody recognition of the Tyr397 autophosphorylation site of FAK (pFAK-Tyr 397), was observed on the vessels of WT mice that were undergoing remodeling, but the level was substantially lower in Angpt $2^{i \Delta / \Delta}$ mice (Figure 9, F and G). The integrin dependence of Angpt2-induced FAK phosphorylation was further confirmed by knockdown of integrin $\alpha_{5}$ or integrin $\beta_{1}$ from HUVECs, which led to diminished Angpt2-induced FAK phosphorylation (Figure 9H). Given that FAK significantly contributes to formation of abnormal 
A
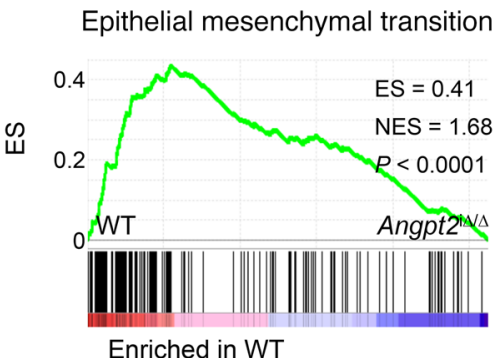

GO proteinaceous extracellular matrix

Enriched in WT
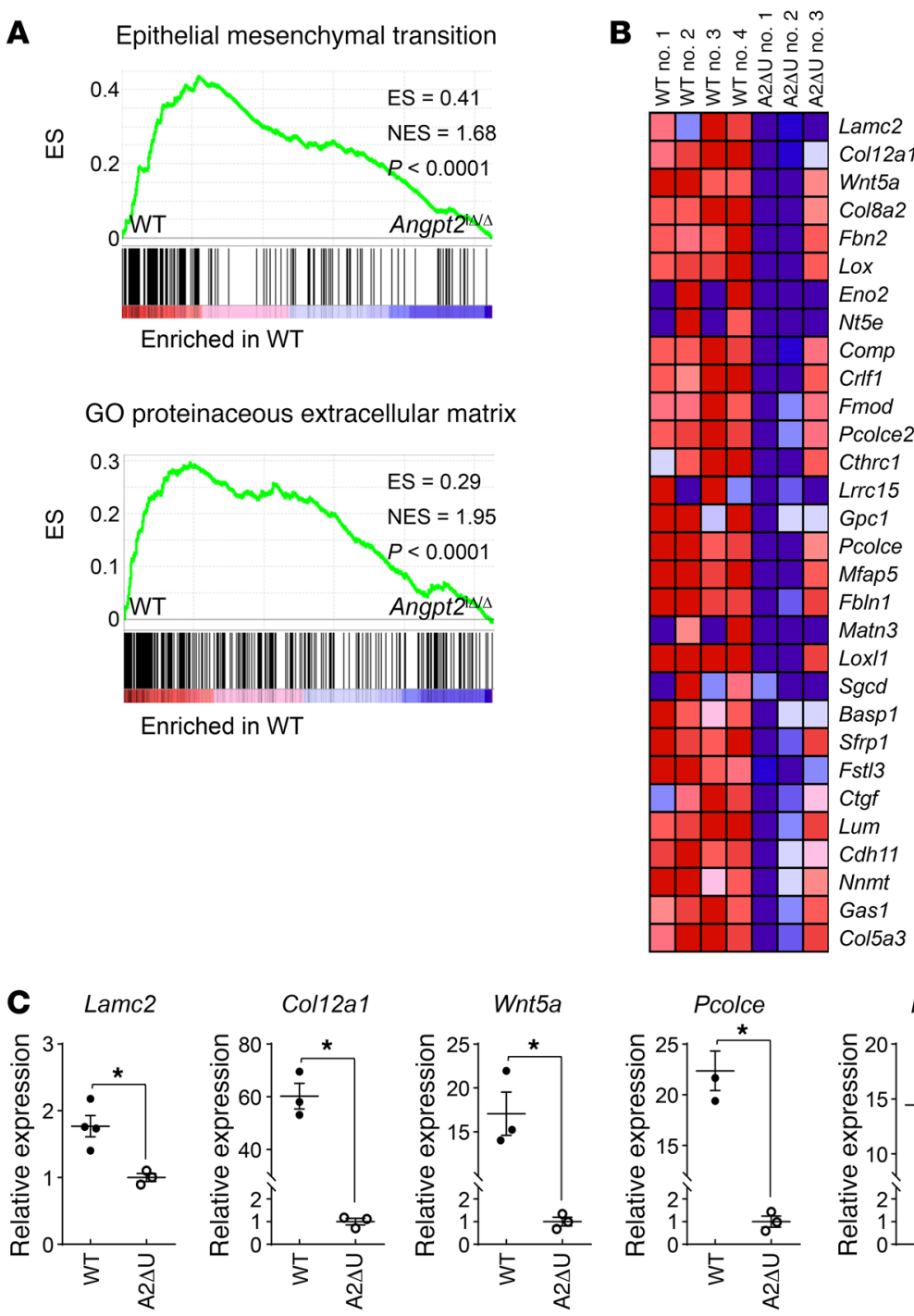

vasculature around the tumor by increasing EC migration and permeability (49), these data imply that Angpt2 promotes adverse vascular remodeling through integrin $\alpha_{5} \beta_{1}$ binding and FAK phosphorylation in the ischemic heart.

To further evaluate the impact of Angpt2 on chronic vascular remodeling, we performed gene expression profiling on ECs of the border zone that were freshly isolated from infarcted hearts using $3^{\prime}$ mRNA sequencing $(50,51)$. Intriguingly, gene set expression analysis (GSEA) showed the downregulation of genes related to the epithelial mesenchymal transition (EMT) in the ECs of Angpt ${ }^{2 / / 4}$ mice relative to those of WT mice (Figure 10, A and B). mRNA expression of the genes (Col12a1, Wnt5a, Lamc2, Fstl3, Pcolce) involved in endothelial-to-mesenchymal transition (EndoMT) were also significantly decreased in the heart ECs of Angpt $2^{i / /}$ mice compared with those of WT mice (Figure 10C and Supplemental Table 1). The EndoMT is a specialized form of EMT that is characterized by the loss of endothelial identity and the acquisition of mesenchymal and stem-like characteristics by ECs (52). Notably, EndoMT contributes to the disintegration of the vascular barrier and to abnormal vascular remodeling of the brain microvasculature (53), which are similar to the vascular changes
Figure 10. Genetic depletion of Angpt2 mitigates expression of EndoMT-related genes. Adult WT or Angpt $2^{i / /}(\mathrm{A} 2 \Delta \mathrm{U})$ mice were subject to $\mathrm{Ml}$, and the infarct border zone ECs were freshly isolated at 2 weeks after MI. (A) GSEA of isolated ECs at the border zone showing downregulation of the genes of EMT signature and GO terms proteinaceous ECM in Angptz $2^{i / 4}$ mice compared with WT mice. ES, enrichment score. NES, normalized enrichment score. $n=3-4$, each group. (B) Heatmap of EMT signature genes of ECs sorted from WT and Angpt $2^{i / / 4}$ mice. (C) The relative expression level of each EndoMT-related gene was confirmed by quantitative PCR ( $n=3$, each group). ${ }^{*} P<0.05$ versus WT, Mann-Whitney $U$ test. Error bars represent mean \pm SD.

observed in our study. Furthermore, the GSEA data revealed a decreased association with the GO term proteinaceous ECM in Angpt $2^{i / 4}$ mice compared with WT mice (Figure 10A), which is a resultant phenotype of EndoMT. These GSEA findings support the notion that Angpt2 plays a dominant role in exacerbating adverse vascular remodeling after MI.

Genetic or pharmacological inhibition of Angpt2 ameliorates postischemic cardiovascular remodeling. Finally, we asked whether genetic depletion or pharmacological inhibition of Angpt2 could mitigate infarct progression and consequent heart failure. Indeed, pericyte detachment, RBC leakage, heparanase expression, leukocyte infiltration, proinflammatory macrophage polarization, and vascular adverse remodeling were all effectively mitigated by anti-Angpt2 antibody treatment (23) (Figure 11, $\mathrm{A}$ and $\mathrm{B})$. Furthermore, antibody-mediated neutralization of Angpt2 suppressed the excessive activation of integrin $\beta_{1}$ in the infarct border ECs, reinforcing our suggestion that Angpt2 activates integrin $\alpha_{5} \beta_{1}$ after MI (Supplemental Figure 12). Consequently, global depletion of Angpt2 or treatment with an anti-Angpt 2 blocking antibody 6 hours after MI (Figure 12A) attenuated infarct progression by approximately $57 \%$ and approximately $43 \%$ at 3 weeks after MI, respectively (Figure 12, B and C). Cardiac fibrosis, as measured by fibronectin deposition 3 weeks after MI, was also consistently lower in Angpt $2^{i / / 4}$ mice $(\sim 60 \%)$ and in anti-Angpt2 antibody-treated mice $(\sim 42 \%)$ (Figure 12, D and E).

To determine whether Angpt2 depletion/inhibition had a protective effect on cardiac function, we measured the left ventricular systolic function by echocardiography 3 weeks after MI (Figure 12F). Intriguingly, genetic depletion of Angpt2 or anti-Angpt2 antibody treatment effectively mitigated the decrease in ejection fraction ( $49 \%$ and $\sim 33 \%)$, stroke volume $(\sim 48 \%$ and $\sim 37 \%)$, and cardiac output ( $49 \%$ and $\sim 36 \%$ ), respectively (Figure $12, \mathrm{~F}$ and G).

We also investigated whether Angpt2 plays a crucial role in cardiac ischemia/reperfusion (I/R) using a mouse model. Similarly to the spatiotemporal expression pattern in MI, distinct FOXO1 nuclear localization and Angpt2 expression by ECs was observed in the 


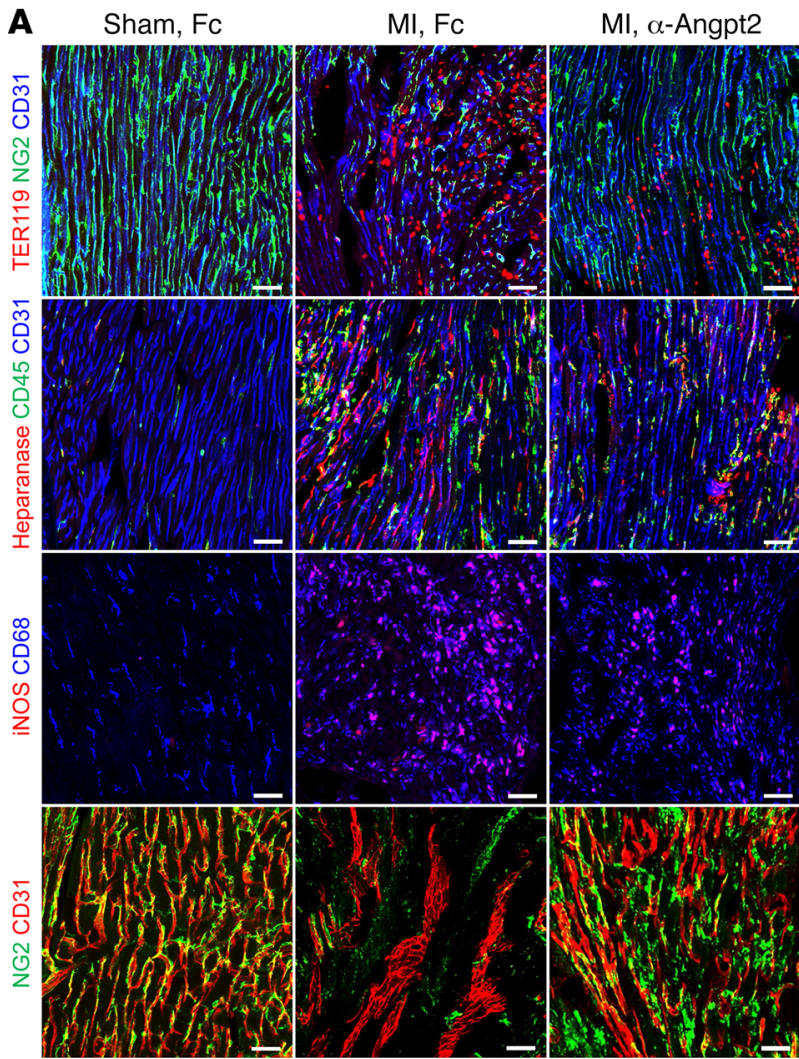

B
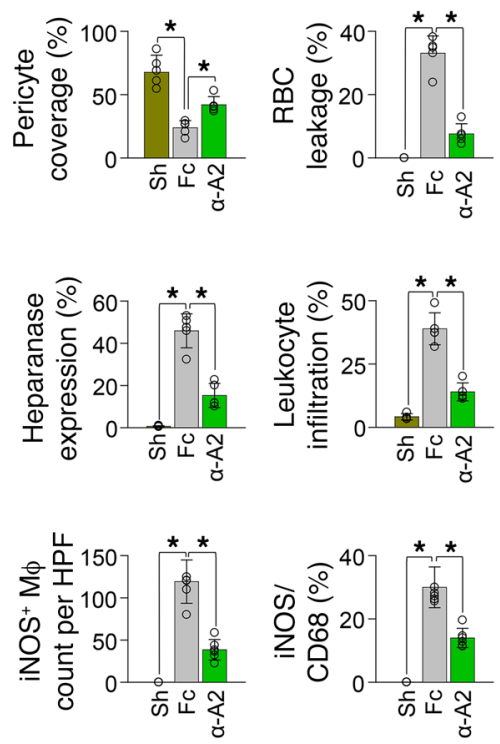

Figure 11. Therapeutic inhibition of Angpt2 prevents vascular disintegration, permeability, inflammation, adverse remodeling, and proinflammatory macrophage polarization after $\mathrm{MI}$. Fc or $\alpha$-Angpt2 ( $\alpha$-A2) $(20 \mathrm{mg} / \mathrm{kg}$, intraperitoneally) was administered to WT mice at 6 hours after MI or sham procedure, followed by repeated injection of the same dose at 1 week intervals. (A and $\mathbf{B}$ ) Images and comparisons of $\mathrm{NG2}^{+}$ pericyte coverage, TER119+ RBC leakage, heparanase expression, $\mathrm{CD}_{4} 5^{+}$leukocytes infiltration at 3 days after, iNOS+ macrophage count and proportion at 7 days after, and $\mathrm{NG2}^{+}$pericyte coverage at 14 days after MI. $n=5-6$, each group. Scale bars: $50 \mu \mathrm{m}$. ${ }^{*} P<$ 0.025 , Kruskal-Wallis test followed by Mann-Whitney $U$ test for post hoc pairwise comparisons. Error bars represent mean $\pm \mathrm{SD}$. reperfused area starting 2 days after the I/R injury (Supplemental Figure 13, A-D). Moreover, Angpt2 substantially contributed to vascular barrier disruption, vascular inflammation, proinflammatory macrophage polarization, and adverse vascular remodeling after I/R injury, and this was markedly alleviated by anti-Angpt2 antibody treatment (Supplemental Figure 13, E and F). Consequently, antiAngpt2 antibody treatment greatly mitigated left ventricular wall thinning and infarct progression after I/R injury (Figure 12, H-J).

\section{Discussion}

This study demonstrated that Angpt2 plays crucial roles in EC destabilization and remodeling in the infarct border zone after MI and provided insights into the contribution of Angpt 2 to the proinflammatory milieu (Figure 13). Intriguingly, EC-specific deletion of Angpt2 preserved endothelial barrier integrity and quiescence after MI; this led to improved cardiac oxygenation and marked reduction of the infarct area in the acute phase of MI. Moreover, global deletion of Angpt 2 mitigated proinflammatory macrophage polarization and adverse vascular remodeling in the chronic remodeling phase. It was especially noteworthy that genetic or antibody-mediated pharmacological inhibition of Angpt2 markedly ameliorated cardiac hypoxia and inflammation and the consequent progression of heart failure.

This study demonstrates the distinct spatiotemporal expression profile of Angpt2 after MI and I/R, which, to date, has been obscure due to a lack of reliable antibodies (54). Using our recently developed anti-Angpt2 antibody (23), which is highly sensitive and specific to Angpt2, we demonstrate distinct expressions of Angpt2 on the ECs and proinflammatory macrophages after ischemia. This prompted us to further investigate the role of
Angpt2 in postischemic cardiovascular remodeling. We recently reported that the FOXO1/Angpt2 axis suppresses the endothelial PI3K/Akt-signaling pathway, the main downstream pathway for Tie2 that plays a vital role in maintaining vascular integrity, inducing vascular destabilization in a positive feedback manner (25), and that it is central to the pathogenesis of sepsis (23), diabetic retinopathy (25), and tumor vessel destabilization (38). This study provides additional insights into the serious detrimental effects of the FOXO1/Angpt2 axis in exacerbating cardiac hypoxia and infarct progression by impairing effective perfusion of the ischemic border.

Our study also highlights the substantial role of Angpt2induced vascular inflammation in cardiovascular remodeling. In quiescent ECs, Angpt1/Tie2 signaling recruits an intracellular protein, A2O-binding inhibitor of NF- $\mathrm{kB}$ activation 2 (ABIN2), which strongly inhibits the NF-kB pathway $(25,36)$, whereas Angpt2 activates the NF-kB pathway to further aggravate vascular inflammation and augment adhesion molecule expression (17, 55). Accordingly, this study demonstrated that Angpt2 substantially contributes to the upregulation of NF- $\mathrm{kB}$ p 65 expression in ECs of the border zone and increases the expression of heparanase and adhesion molecules, leading to robust infiltration of inflammatory cells and disorganization/degradation of eGC and cECM. Furthermore, our data show the preferential expression of Angpt 2 by proinflammatory macrophages and the colocalization of Angpt2 with a putative receptor, integrin $\alpha_{5} \beta_{1}$, and its downstream effector, pERK. Previous studies have highlighted the crucial role of the ERK pathway in determining the characteristics of proinflammatory macrophages $(46,56)$. Here, we show that genetic deletion or pharmacological inhibition of Angpt2/ 
A

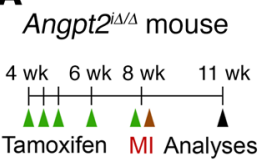

WT mouse

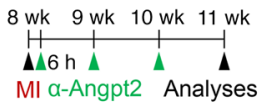

Ml a-Angpt2 Analyses
B

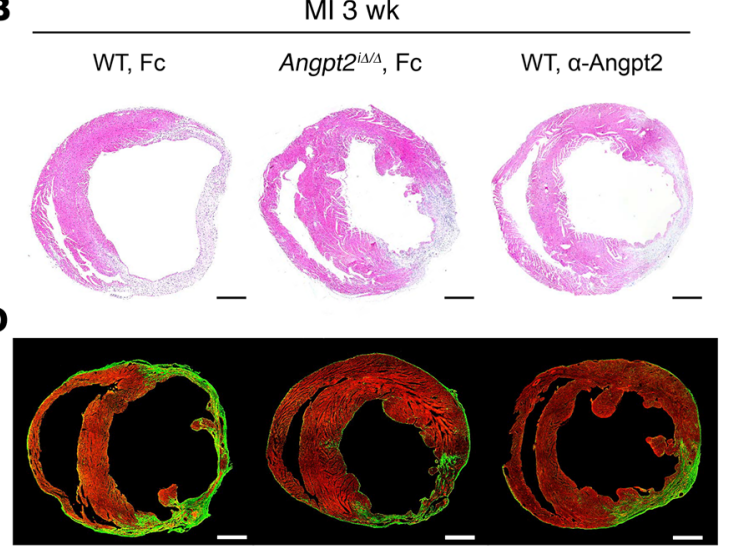

Fibronectin CD31
C

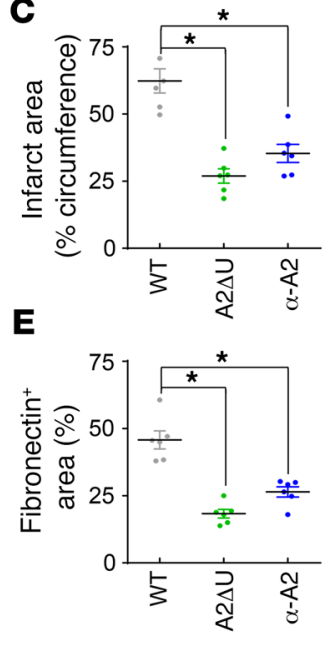

$\mathbf{F}$
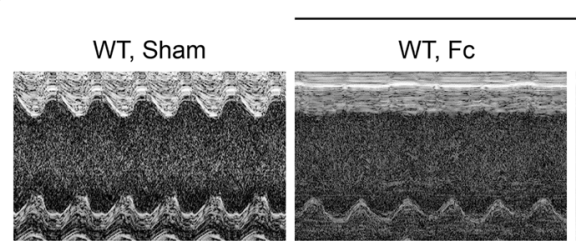

MI 3 wk
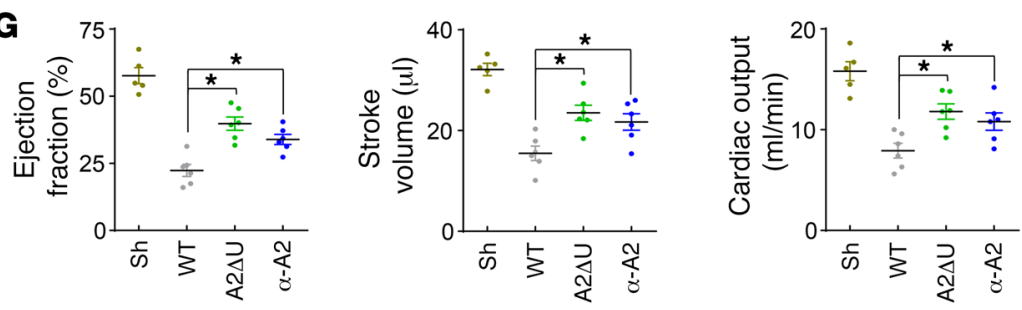

H

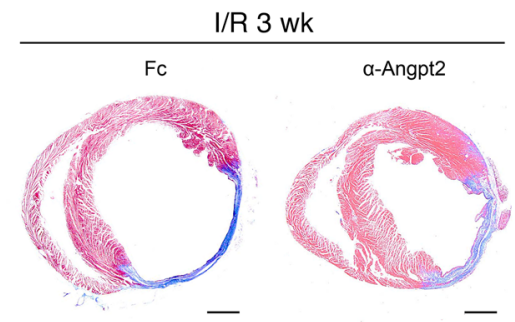

I

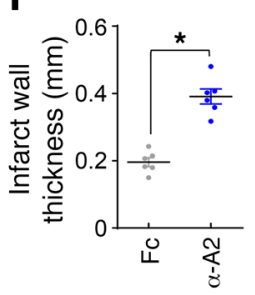

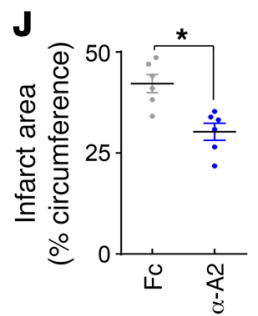

Figure 12. Genetic deletion or blocking antibody of Angpt2 ameliorates infarct size and adverse cardiac remodeling after myocardial ischemia. Adult WT or Angpt $2^{i \Delta / \Delta}$ mice were subject to MI, I/R, or sham procedure, and hearts were analyzed at 3 weeks after the procedure. (A) Diagram depicting preparation of animals and experimental schedules. Fc or $\alpha$-Angpt2 $\left(20 \mathrm{mg} / \mathrm{kg}\right.$, intraperitoneally) was administered to WT or Angpt $2^{i \Delta / \Delta}$ mice at 6 hours after MI, followed by repeated injections of the same dose at 1-week intervals. (B and C) Images and comparisons of infarction area. $n=5-6$, each group. Scale bars: $1 \mathrm{~mm}$. ( $\mathbf{D}$ and $\mathbf{E}$ ) Images and comparisons of cardiac fibrosis represented by fibronectin ${ }^{+}$area. $n=6$, each group. Scale bars: $1 \mathrm{~mm}$. (F and $\left.\mathbf{G}\right)$ Images and comparisons of cardiac systolic function evaluated by echocardiography. $n=6$, each group. (C, E, and $\mathbf{G})$ Comparisons of indicated parameters. ${ }^{*} P<$ 0.025 , Kruskal-Wallis test followed by Mann-Whitney $U$ test for post hoc pairwise comparisons. Significance was adjusted for multiple comparisons using Bonferroni's method. (H-J) Fc or $\alpha$-Angpt2 (20 mg/kg, intraperitoneally) was administered to WT mice at 6 hours after I/R, followed by repeated injections of the same dose at 1 week intervals. Images of cardiac fibrosis determined by Masson trichrome stain and comparisons of indicated parameters. $n=6$, each group. Scale bars: $1 \mathrm{~mm}$. ${ }^{*} P<0.05$ versus WT, Mann-Whitney $U$ test. Error bars represent mean \pm SD.

integrin $\alpha_{5} \beta_{1}$ signaling decreases ERK phosphorylation and proinflammatory macrophage polarization, in accordance with the findings of an in vitro study (57), which strongly suggests that Angpt2 elicits ERK phosphorylation and proinflammatory macrophage polarization after myocardial ischemia. Collectively, these results suggest that Angpt2 plays an integral role in establishing an inflammatory cardiac milieu by augmenting neutrophil infiltration and proinflammatory macrophage polarization.
Previous reports suggest the critical roles of the Angpt-Tie2 system in vascular remodeling $(34,55)$ and tumor angiogenesis (38), but the precise role of Angpt2 in vascular remodeling in ischemic organs is not fully understood. This study demonstrates that Angpt2 promotes adverse vascular remodeling in ischemic hearts via the Angpt2/integrin $\alpha_{5} \beta_{1} /$ FAK-signaling pathway. We note that the infarct border vessels with disintegrated morphology highly expressed pFAK-Tyr 397 as well as Angpt2. FAK is a multifacet- 


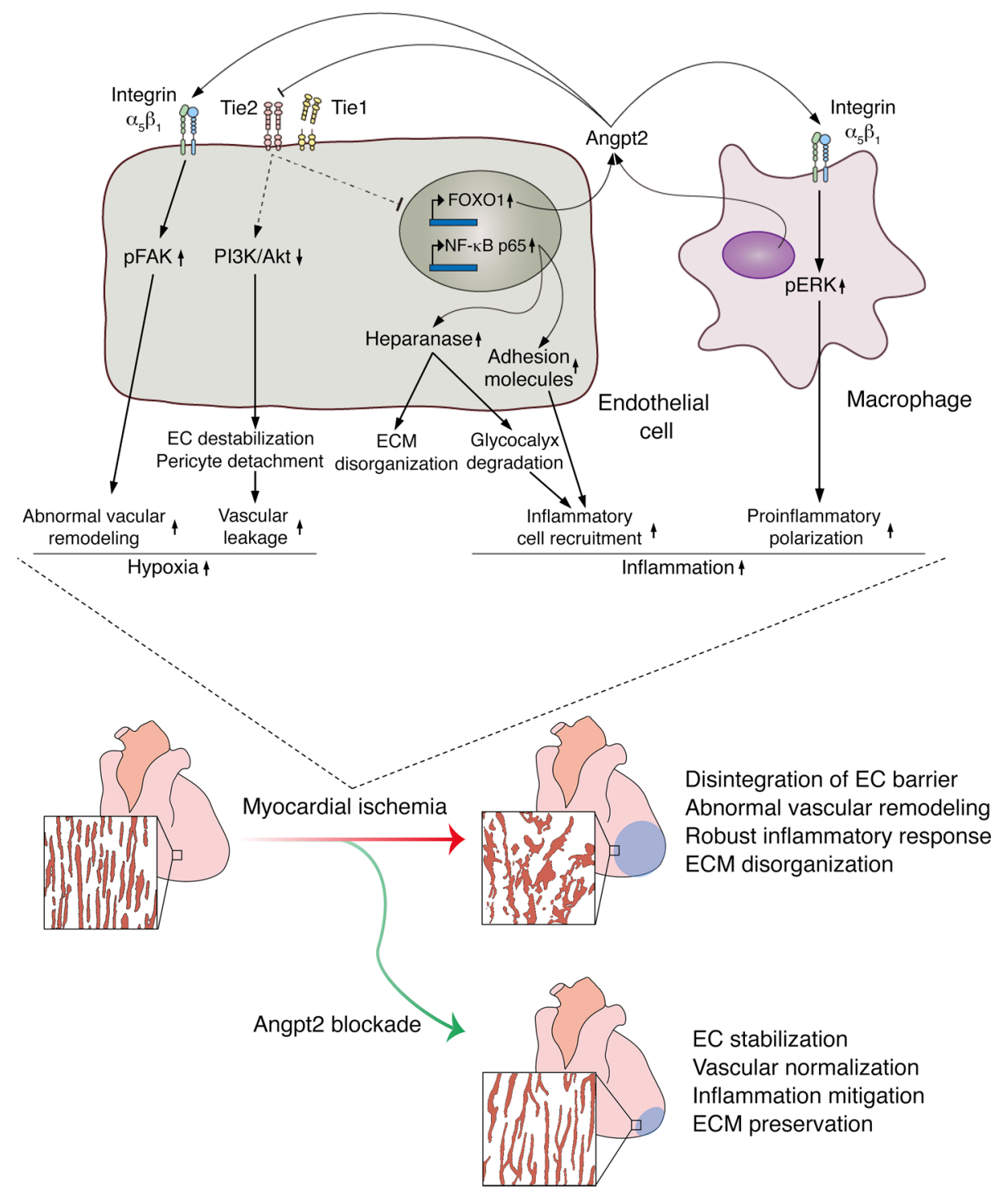

Figure 13. Schematic diagram depicting the roles of Angpt2 in exacerbating cardiac hypoxia and inflammation after myocardial ischemia. Myocardial ischemia induces disintegration of endothelial barrier, abnormal vascular remodeling, robust inflammatory responses, and ECM disorganization, and these pathological changes can be effectively mitigated by Angpt2 blockade. Mechanistically, in ECs, the increase in Angpt2 expression is directly regulated by FOX01, which antagonizes Tie2 signaling and consequently attenuates PI3K/Akt signaling and enhances NF- $\kappa B$ p 65 and FOX01 expression. Thus, increased FOXO1 transcriptional activity forms a positive feedback loop with Angpt2 and exerts a sustained effect on ECs, leading to destabilization and remodeling. Pericyte detachment and EC destabilization presumably due to attenuated PI3K/Akt signaling exacerbate vascular leakage. Enhanced activity of NF- $\kappa B$ p 65 augments endothelial expression of heparanase and adhesion molecules, further promoting inflammatory cell recruitment and ECM disorganization. In addition, Angpt2/integrin $\alpha_{5} \beta_{1}$ signaling promotes abnormal vascular remodeling through FAK phosphorylation, resulting in chronic hypoxia. In macrophages, the Angpt2/ integrin $\alpha_{5} \beta_{1} /$ ERK-signaling pathway plays an important role in proinflammatory macrophage polarization in autocrine and paracrine manners. These exacerbating roles of Angpt2 in cardiac hypoxia and inflammation after myocardial ischemia eventually trigger deterioration of cardiac structure and function, leading to heart failure.

ed regulator of intracellular signaling that is involved in processes such as cell motility, survival, and proliferation in various tumor cells and in the surrounding microenvironment components, including ECs (49). Indeed, FAK plays a critical role in vascular remodeling, and orthotropic glioma implantation in Fak ${ }^{i \triangle E C}$ mice results in tumor vessel normalization (58), similar to the normalized morphology of the vessels in the infarct border area in Ang$p t 2^{i / / 4}$ mice. Together with normalization of vascular morphology, Angpt2 deletion led to marked reduction of pFAK-Tyr 397 expression in infarct border area vessels at the chronic remodeling phase, reinforcing the idea that Angpt2/integrin $\alpha_{5} \beta_{1} /$ FAK signaling is the main driver of vascular remodeling in ischemic conditions.

The EndoMT is a fundamental step in the development of cardiac valves (59), and interestingly, the emergence of cardiac pericytes and vascular smooth muscles cells derived from ECs through EndoMT has also been recognized (60). Moreover, a recent study (61) showed that Angpt2 induces cerebral cavernous malformation through the EndoMT, and this was effectively normalized by an anti-Angpt2 blocking antibody. In accordance with these studies, our GSEA findings strongly suggest that the EndoMT serves as a putative mechanism for Angpt2-induced vascular remodeling, and this is supported by the well-recognized augmenting role of FAK signaling in EMT/EndoMT progression (49).

Myocardial ischemia and the subsequent reperfusion induced by percutaneous coronary intervention or cardiac surgery after MI elicit a robust inflammatory response and cause additional damage to the heart, despite their myocardial salvaging effects $(62,63)$. In this regard, mitigating inflammation and reperfusion injury after $\mathrm{MI}$ and subsequently reducing infarct size are the primary goals for preventing the initiation and progression of ischemic heart failure $(39,62)$. Importantly, the expression and the deleterious effects of Angpt2, which augmented cardiac hypoxia and inflammation, were recapitulated in an I/R model, suggesting the integral role of Angpt 2 in the pathogenesis of postischemic cardiovascular remodeling. While a previous report has shown that 3-hydroxy-3-methylglutaryl-CoA reductase inhibitors may suppress the FOXO1/ Angpt2 axis through in vitro drug screening (64), the exact mechanism remains unclear and the effects are yet to be demonstrated in vivo. Meanwhile, in our study, the antibody-mediated inhibition of Angpt2 considerably abrogated Angpt2-induced vascular disin- 
tegration, vascular inflammation, proinflammatory macrophage polarization and adverse vascular remodeling, even when the antibody was injected 6 hours after MI or I/R to mimic a clinically relevant situation. Further, in line with evidence that hypoxia and inflammation play an important part in triggering and promoting tissue fibrosis (65), our findings highlight that pharmacological inhibition of Angpt2 markedly ameliorates cardiac fibrosis and decline in cardiac function after myocardial ischemia. These findings provide mechanistic insights that could enormously expand the therapeutic repertoire for ischemic heart disease.

Together, the results of this study elucidate the integral roles of Angpt2 in the pathogenesis of postischemic cardiovascular remodeling and designate Angpt2 as a promising therapeutic target, in which inhibition of Angpt 2 markedly ameliorates cardiac hypoxia and inflammation and subsequently prevents ischemic heart failure.

\section{Methods}

Mice. Specific pathogen-free (SPF) C57BL/6J mice, Tie2-GFP mice, and $U B C-C r e-E^{T 2}$ mice were purchased from the Jackson Laboratory. Angpt2-EGFP mice (Tg [Angpt2-EGFP] DJ90Gsat/Mmucd) were purchased from the Mutant Mouse Regional Resource Center. Angpt1GFP mice (29) were provided by Sean Morrison (University of Texas Southwestern Medical Center, Dallas, Texas, USA). Foxo $1^{f / f l}$ mice were provided by Ronald A. DePinho (University of Texas MD Anderson Cancer Center, Houston, Texas, USA). VE-cadherin-Cre-ER ${ }^{\mathrm{T} 2}$ (28),

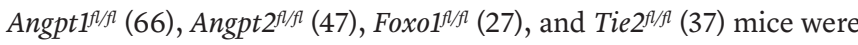
transferred and bred in our SPF animal facility at KAIST. To deplete Angpt2, Foxo1, or Tie2 genes specifically in ECs, VE-cadherin-Cre$\mathrm{ER}^{\mathrm{T} 2}$ mice were mated with either Angpt $2^{f / f l}$, Foxo1 $1^{f / f l}$, or Tie $2^{f / f l}$ mice to obtain EC-specific Angpt2-, Foxo1-, or Tie2-deleted mice, respectively, in a tamoxifen-dependent manner. To delete Angpt1 or Angpt2 globally, Angpt $1^{f / f l}$ or Angpt $2^{f / f l}$ mice were crossed with the UBC-Cre-ER ${ }^{\mathrm{T} 2}$ mice. Tamoxifen ( $2 \mathrm{mg}$, T5648; Sigma-Aldrich) was injected intraperitoneally a total of 3 times every 2 days into the 4 -week-old genetically modified mice, followed by an additional injection $(2 \mathrm{mg}) 2$ weeks later. In the case of global deletion, considering the rapid turnover rate of macrophage life span of 10 to 14 days (67), tamoxifen ( $2 \mathrm{mg}$ ) was additionally given immediately before the procedure. Cre-ER ${ }^{\mathrm{T} 2}$-positive but flox/flox-negative mice among the littermates for each experiment were defined as WT mice.

Surgical procedures. Eight- to ten-week old male C57BL/6J WT or genetically modified mice were subjected to an MI or I/R injury model. Mice were anesthetized with isoflurane and intubated using a 20 -gauge intravenous catheter with a blunt end and ventilated with a mixture of oxygen and isoflurane (1.5\%-2.0\%). The chest cavity was opened in the third intercostal space, and permanent suture by 8-0 prolene was made for the MI procedure. Complete occlusion of the left anterior descending (LAD) artery was confirmed by the presence of myocardial blanching. For the I/R injury model, a 7-0 prolene suture was made with a section of PE-10 tubing placed over the LAD artery, $1 \mathrm{~mm}$ below the tip of the left atrium. After occlusion for 30 minutes, the ligature was released by removing PE-10 tubing for reperfusion of LAD. After permanent or temporary ligation of LAD, the chest wall was closed with 6-0 silk suture. Sham-operated mice underwent the same procedure without coronary artery ligation. Animals were not randomized; however, procedures were performed by researchers blinded to the genotype and drug- or antibody-treatment group.

For more information, see Supplemental Methods.

Statistics. Values are presented as mean \pm SD. Statistical differences between the means were compared by the 2-sided Mann-Whitney $U$ test for 2 groups and by the Kruskal-Wallis test followed by Bonferroni's correction for multiple groups. Statistical analysis was performed with PASW Statistics 18 (SPSS). Statistical significance was set to a $P$ value of less than 0.05 .

Study approval. Animal care and experimental procedures were approved by the Animal Care Committee of KAIST (no. KA2013-40). Mice were housed in accordance with the Institutional Animal Care and Use Committee regulations at KAIST.

\section{Author contributions}

SJL and GYK designed the research studies. SJL, CKL, SK, IP, YHK, SKK, SPH, and HB conducted the experiments and analyzed the data. YH and YK provided the mice, and SJL and GYK generated the figures and wrote and edited the manuscript.

\section{Acknowledgments}

We thank Hyun-Tae Kim for careful management for the animal facility and mice. We thank Jeomil Bae and Mijeong Kim for the antibody work. This study was supported by the Institute of Basic Science (IBS-R025-D1-2015 to GYK) funded by the Ministry of Science, ICT, and Future Planning, South Korea.

Address correspondence to: Gou Young Koh, Center for Vascular Research, IBS; Graduate School of Medical Science and Engineering, KAIST, 291 Daehak-ro, Daejeon 34141, South Korea. Phone: 82.42.350.2638; Email: gykoh@kaist.ac.kr.

SJL's present address is: Division of Cardiology, Severance Cardiovascular Hospital, Yonsei University College of Medicine, Seoul, South Korea.
1. World Health Organization. The top 10 causes of death. http://www.who.int/mediacentre/ factsheets/fs310/en/. WHO website. Published May 24, 2018. Accessed September 25, 2018.

2. Braunwald $\mathrm{E}$. The war against heart failure: the Lancet lecture. Lancet. 2015;385(9970):812-824.

3. Sano M, et al. p53-induced inhibition of Hif-1 causes cardiac dysfunction during pressure overload. Nature. 2007;446(7134):444-448.

4. Taimeh Z, Loughran J, Birks EJ, Bolli R. Vascular endothelial growth factor in heart failure. Nat Rev Cardiol. 2013;10(9):519-530.
5. Ylä-Herttuala S, Rissanen TT, Vajanto I, Hartikainen J. Vascular endothelial growth factors: biology and current status of clinical applications in cardiovascular medicine. J Am Coll Cardiol. 2007;49(10):1015-1026.

6. Weis SM, Cheresh DA. Pathophysiological consequences of VEGF-induced vascular permeability Nature. 2005;437(7058):497-504.

7. Paul R, et al. Src deficiency or blockade of Src activity in mice provides cerebral protection following stroke. Nat Med. 2001;7(2):222-227.

8. Weis S, et al. Src blockade stabilizes a Flk/ cadherin complex, reducing edema and tissue injury following myocardial infarction. JClin Invest. 2004;113(6):885-894.

9. Pereira ER, Frudd K, Awad W, Hendershot LM. Endoplasmic reticulum (ER) stress and hypoxia response pathways interact to potentiate hypoxiainducible factor 1 (HIF-1) transcriptional activity on targets like vascular endothelial growth factor (VEGF). J Biol Chem. 2014;289(6):3352-3364.

10. Augustin HG, Koh GY. Organotypic vasculature: From descriptive heterogeneity to functional pathophysiology. Science. 
2017;357(6353):eaal2379.

11. Felcht $\mathrm{M}$, et al. Angiopoietin-2 differentially regulates angiogenesis through TIE2 and integrin signaling. J Clin Invest. 2012;122(6):1991-2005.

12. Saharinen P, et al. Angiopoietins assemble distinct Tie2 signalling complexes in endothelial cell-cell and cell-matrix contacts. Nat Cell Biol. 2008;10(5):527-537.

13. Kim KE, Cho CH, Kim HZ, Baluk P, McDonald $\mathrm{DM}, \mathrm{Koh}$ GY. In vivo actions of angiopoietins on quiescent and remodeling blood and lymphatic vessels in mouse airways and skin. Arterioscler Thromb Vasc Biol. 2007;27(3):564-570.

14. Saharinen P, Eklund L, Alitalo K. Therapeutic targeting of the angiopoietin-TIE pathway. Nat Rev Drug Discov. 2017;16(9):635-661.

15. Kim J, et al. Impaired angiopoietin/Tie2 signaling compromises Schlemm's canal integrity and induces glaucoma. J Clin Invest. 2017;127(10):3877-3896

16. Kenig-Kozlovsky $Y$, et al. Ascending vasa recta are angiopoietin/Tie2-dependent lymphatic-like vessels. JAm Soc Nephrol. 2018;29(4):1097-1107.

17. Fiedler U, et al. Angiopoietin-2 sensitizes endothelial cells to TNF-alpha and has a crucial role in the induction of inflammation. Nat Med. 2006;12(2):235-239.

18. Hakanpaa L, et al. Endothelial destabilization by angiopoietin- 2 via integrin $\beta 1$ activation. Nat Commun. 2015;6:5962.

19. Lorbeer R, et al. Circulating angiopoietin-2, its soluble receptor Tie-2, and mortality in the general population. Eur J Heart Fail. 2013;15(12):1327-1334.

20. Eleuteri E, et al. Stepwise increase of angiopoietin-2 serum levels is related to haemodynamic and functional impairment in stable chronic heart failure. Eur JCardiovasc Prev Rehabil. 2011;18(4):607-614.

21. Link A, et al. Circulating angiopoietins and cardiovascular mortality in cardiogenic shock. Eur Heart J. 2013;34(22):1651-1662.

22. Pöss J, et al. Angiopoietin- 2 in acute myocardial infarction complicated by cardiogenic shock--a biomarker substudy of the IABP-SHOCK II-Trial. Eur J Heart Fail. 2015;17(11):1152-1160.

23. Han S, et al. Amelioration of sepsis by TIE2 activation-induced vascular protection. Sci Transl Med. 2016;8(335):335ra55.

24. Tian F, Liang PH, Li LY. Inhibition of endothelial progenitor cell differentiation by VEGI. Blood. 2009;113(21):5352-5360.

25. Park DY, et al. Plastic roles of pericytes in the blood-retinal barrier. Nat Commun. 2017;8:15296.

26. Shao D, et al. A functional interaction between Hippo-YAP signalling and FoxO1 mediates the oxidative stress response. Nat Commun. 2014;5:3315.

27. Paik JH, et al. FoxOs are lineage-restricted redundant tumor suppressors and regulate endothelial cell homeostasis. Cell. 2007;128(2):309-323.

28 . Okabe K, et al. Neurons limit angiogenesis by titrating VEGF in retina. Cell. 2014;159(3):584-596.

29. Zhou BO, Ding L, Morrison SJ. Hematopoietic stem and progenitor cells regulate the regeneration of their niche by secreting Angiopoietin-1.
Elife. 2015;4:e05521.

30. Augustin HG, Koh GY, Thurston G, Alitalo K. Control of vascular morphogenesis and homeostasis through the angiopoietin-Tie system. Nat Rev Mol Cell Biol. 2009;10(3):165-177.

31. Kenig-Kozlovsky Y, et al. Ascending vasa recta are angiopoietin/Tie2-dependent lymphatic-like vessels. J Am Soc Nephrol. 2018;29(4):1097-1107.

32. Thomson BR, et al. Angiopoietin-1 is required for Schlemm's canal development in mice and humans. JClin Invest. 2017;127(12):4421-4436.

33. D’Amico G, et al. Tie1 deletion inhibits tumor growth and improves angiopoietin antagonist therapy. JClin Invest. 2014;124(2):824-834.

34. Korhonen EA, et al. Tie1 controls angiopoietin function in vascular remodeling and inflammation. J Clin Invest. 2016;126(9):3495-3510.

35. Kim M, et al. Opposing actions of angiopoietin-2 on Tie2 signaling and FOXO1 activation. JClin Invest. 2016;126(9):3511-3525.

36. Ziegler T, et al. Angiopoietin 2 mediates microvascular and hemodynamic alterations in sepsis. JClin Invest. 2013;123(8):3436-3445.

37. Savant $S$, et al. The orphan receptor Tie1 controls angiogenesis and vascular remodeling by differentially regulating Tie 2 in tip and stalk cells. Cell Rep. 2015;12(11):1761-1773.

38. Park JS, et al. Normalization of tumor vessels by Tie2 activation and Ang2 inhibition enhances drug delivery and produces a favorable tumor microenvironment. Cancer Cell. 2016;30(6):953-967.

39. Seropian IM, Toldo S, Van Tassell BW, Abbate A. Anti-inflammatory strategies for ventricular remodeling following ST-segment elevation acute myocardial infarction. J Am Coll Cardiol. 2014;63(16):1593-1603.

40. Andela VB, Schwarz EM, Puzas JE, O'Keefe RJ, Rosier RN. Tumor metastasis and the reciprocal regulation of prometastatic and antimetastatic factors by nuclear factor kappaB. Cancer Res. 2000;60(23):6557-6562.

41. Hadigal SR, et al. Heparanase is a host enzyme required for herpes simplex virus-1 release from cells. Nat Commun. 2015;6:6985.

42. Liddiard K, Taylor PR. Understanding local macrophage phenotypes in disease: shape-shifting macrophages. Nat Med. 2015;21(2):119-120.

43. Nahrendorf M, Swirski FK. Monocyte and macrophage heterogeneity in the heart. Circ Res. 2013;112(12):1624-1633.

44. Shiraishi M, et al. Alternatively activated macrophages determine repair of the infarcted adult murine heart. JClin Invest. 2016;126(6):2151-2166.

45. Fang HY, et al. Hypoxia-inducible factors 1 and 2 are important transcriptional effectors in primary macrophages experiencing hypoxia. Blood. 2009;114(4):844-859.

46. Zhu L, et al. TSC1 controls macrophage polarization to prevent inflammatory disease. Nat Commun. 2014;5:4696.

47. Shen B, et al. Genetic dissection of tie pathway in mouse lymphatic maturation and valve development. Arterioscler Thromb Vasc Biol. 2014;34(6):1221-1230.

48. Clausen BE, Burkhardt C, Reith W, Renkawitz $\mathrm{R}$, Förster I. Conditional gene targeting in mac- rophages and granulocytes using LysMcre mice. Transgenic Res. 1999;8(4):265-277.

49. Sulzmaier FJ, Jean C, Schlaepfer DD. FAK in cancer: mechanistic findings and clinical applications. Nat Rev Cancer. 2014;14(9):598-610.

50. Moll P, Ante M, Seitz A, Reda T. QuantSeq 3 [prime] mRNA sequencing for RNA quantification. Nat Methods. 2014;11:i-iii.

51. Kasper DM, et al. MicroRNAs establish uniform traits during the architecture of vertebrate embryos. Dev Cell. 2017;40(6):552-565.e5.

52. Kovacic JC, Mercader N, Torres M, Boehm M, Fuster V. Epithelial-to-mesenchymal and endothelial-to-mesenchymal transition: from cardiovascular development to disease. Circulation. 2012;125(14):1795-1808.

53. Maddaluno L, et al. EndMT contributes to the onset and progression of cerebral cavernous malformations. Nature. 2013;498(7455):492-496.

54. Lee KW, Lip GY, Blann AD. Plasma angiopoietin-1, angiopoietin-2, angiopoietin receptor tie-2, and vascular endothelial growth factor levels in acute coronary syndromes. Circulation. 2004;110(16):2355-2360.

55. Potente M, Mäkinen T. Vascular heterogeneity and specialization in development and disease. Nat Rev Mol Cell Biol. 2017;18(8):477-494.

56. Lawrence T, Natoli G. Transcriptional regulation of macrophage polarization: enabling diversity with identity. Nat Rev Immunol. 2011;11(11):750-761.

57. Seok SH, et al. Angiopoietin-1 elicits pro-inflammatory responses in monocytes and differentiating macrophages. Mol Cells. 2013;35(6):550-556.

58. Lee J, Borboa AK, Chun HB, Baird A, Eliceiri BP. Conditional deletion of the focal adhesion kinase FAK alters remodeling of the blood-brain barrier in glioma. Cancer Res. 2010;70(24):10131-10140.

59. Zhang $\mathrm{H}$, et al. Yap1 is required for endothelial to mesenchymal transition of the atrioventricular cushion. J Biol Chem. 2014;289(27):18681-18692.

60. Chen Q, et al. Endothelial cells are progenitors of cardiac pericytes and vascular smooth muscle cells. Nat Commun. 2016;7:12422.

61. Jenny Zhou H, et al. Endothelial exocytosis of angiopoietin-2 resulting from CCM 3 deficiency contributes to cerebral cavernous malformation. Nat Med.2016;22(9):1033-1042.

62. Ibáñez B, Heusch G, Ovize M, Van de Werf F. Evolving therapies for myocardial ischemia/ reperfusion injury. J Am Coll Cardiol. 2015;65(14):1454-1471.

63. Yellon DM, Hausenloy DJ. Myocardial reperfusion injury. N EnglJ Med. 2007;357(11):1121-1135.

64. Ghosh CC, et al. Drug repurposing screen identifies Foxo1-dependent angiopoietin-2 regulation in sepsis. Crit Care Med. 2015;43(7):e230-e240.

65. Wynn TA, Ramalingam TR. Mechanisms of fibrosis: therapeutic translation for fibrotic disease. Nat Med. 2012;18(7):1028-1040.

66. Lee J, et al. Angiopoietin-1 guides directional angiogenesis through integrin $\alpha v \beta 5$ signaling for recovery of ischemic retinopathy. Sci Transl Med. 2013;5(203):203ra127.

67. Geissmann F. The origin of dendritic cells. Nat Immunol. 2007;8(6):558-560. 